\title{
On the Prediction of Solar Cycles
}

\author{
V. Courtillot ${ }^{1}$ D F Fopes ${ }^{1} \cdot$ J.L. Le Mouël ${ }^{1}$
}

Received: 8 September 2020 / Accepted: 28 December 2020 / Published online: 25 January 2021

(C) The Author(s) 2021

\begin{abstract}
This article deals with the prediction of the upcoming solar activity cycle, Solar Cycle 25. We propose that astronomical ephemeris, specifically taken from the catalogs of aphelia of the four Jovian planets, could be drivers of variations in solar activity, represented by the series of sunspot numbers (SSN) from 1749 to 2020. We use singular spectrum analysis (SSA) to associate components with similar periods in the ephemeris and SSN. We determine the transfer function between the two data sets. We improve the match in successive steps: first with Jupiter only, then with the four Jovian planets and finally including commensurable periods of pairs and pairs of pairs of the Jovian planets (following Mörth and Schlamminger in Planetary Motion, Sunspots and Climate, Solar-Terrestrial Influences on Weather and Climate, 193, 1979). The transfer function can be applied to the ephemeris to predict future cycles. We test this with success using the "hindcast prediction" of Solar Cycles 21 to 24, using only data preceding these cycles, and by analyzing separately two 130 and 140 year-long halves of the original series. We conclude with a prediction of Solar Cycle 25 that can be compared to a dozen predictions by other authors: the maximum would occur in 2026.2 ( $\pm 1 \mathrm{yr}$ ) and reach an amplitude of 97.6 ( \pm 7.8 ), similar to that of Solar Cycle 24, therefore sketching a new "Modern minimum", following the Dalton and Gleissberg minima.
\end{abstract}

Keywords Sunspot number · Solar Cycle 25 - Prediction - Singular spectral analysis · Transfer function · Ephemeris of Jovian planets · Commensurability

\section{Introduction}

The series of observations of sunspots is one of the longest available; it is considered as a valuable proxy of solar activity at the centennial time scale. The series is a useful tool to study solar physics, and also the influence of solar variability on space weather, satellite orbits, the well being of astronauts, long distance communications and terrestrial climate,

V. Courtillot

1 Institut de physique du globe de Paris, Geomagnetism and Paleomagnetism, CNRS, Université de Paris, 1 rue Jussieu, 75005 Paris, France 


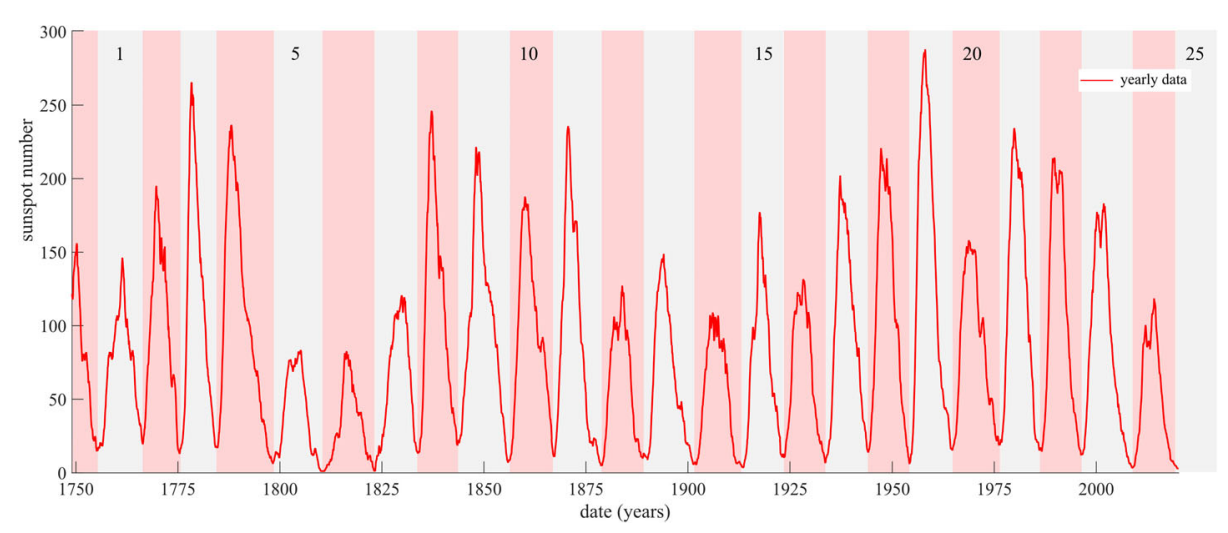

Figure 1 Annual mean sunspot values from January 15, 1749, to March 15, 2020.

to name a few. Mankind is particularly keen to be able to predict the evolution of solar activity and its bearing on space weather and terrestrial climate. Upon a first look at the time series of sunspots (Figure 1), the $\approx 11$ year repetition of an otherwise rather irregular solar cycle, the Schwabe cycle, immediately jumps to the eye. The series is generally considered reliable since the end of the quasi-spotless Maunder minimum (Maunder, 1894; Maunder and Maunder, 1905), that is the early 1700s. We are currently (2020) entering Solar Cycle 25.

The sunspot number series has been revised in 2014-2015 (Clette and Lefèvre, 2016). It is maintained by the Royal Observatory of Belgium at the Sunspot Index and Long-term Solar Observations site (http://www.sidc.be/silso/datafiles).

The topic of sunspot series has generated a vast literature. For accessible and thorough reviews, we refer the general reader to Whitehouse (2020), and the specialists to Vaquero et al. (2016) and Arlt and Vaquero (2020). In 1848, Wolf introduced the sunspot number SSN that now bears his name and in 1852 published his paper showing evidence for a cycle with a period of $\approx 11$ years (see Wolf, 1852). Many scientists have since then tried to predict the duration and amplitude of the cycle to come. As seen in Figure 1, cycles are quite irregular and cannot be predicted using classical extrapolation techniques. Petrovay's (2020) comprehensive review distinguishes three main groups of solar cycle prediction methods: precursor methods that rely on some (often magnetic) measure of solar activity, modelbased methods based on dynamo models, and extrapolation methods based "on the premise that the physical process giving rise to the sunspot number record is statistically homogeneous." Petrovay (2020) concludes that "precursor methods have clearly been superior to extrapolation methods (...). Nevertheless, some extrapolation methods may still be worth further study."

In this paper, we vindicate that last statement. We propose a new way of predicting solar cycles that relies on the analysis of quasi-periodic components of the sunspot series using Singular Spectrum Analysis (SSA; see, e.g., Le Mouël, Lopes, and Courtillot, 2020a). Our method belongs to the third category, with a major addition (see Section 2).

Relying on increasing evidence of an influence of solar activity on the geomagnetic field (e.g. Mayaud, 1972; Currie, 1973; Courtillot and Le Mouël, 1976a, 1976b), some authors tried with moderate success to predict the characteristics of Solar Cycles 23 and 24 (Lantos and Richard, 1998; Duhau, 2003; Svalgaard, Cliver, and Kamide, 2005; Hathaway and Wilson, 2006; Bhatt, Jain, and Aggarwal, 2009). Others started from the observation that there is little or no physical reasoning in the way the Wolf number (SSN, 
which is a pure number) is derived. Some authors worked directly on the SSN (Wilson, 1988; Hathaway, Wilson, and Reichmann, 1994; Li, 1997; Hans Meier, Denkmayr, and Weiss, 1999; Cameron and Schüssler, 2007; Kossobokov, Le Mouël, and Courtillot, 2012; Kossobokov, Le Mouël, and Courtillot, 2016), others on the heliomagnetic field associated with the Schwabe cycle (Schatten et al., 1978; Bushby and Tobias, 2007; Charbonneau, 2014). More recently authors have used data mining combining different kinds of data (e.g. Mwitondi, Raeed, and Yousif, 2012; Li and Zhu, 2013; Pesnell, 2008, 2016).

In the present article, we introduce a "quasi"-mechanism of forcing of solar activity by planetary motions in Section 2, a method of analysis of ephemeris and sunspot series in Section 3. We infer a way to reconstruct and then predict solar activity in Section 4, discuss the results, in particular hindcast some past cycles and divide the series in two halves that are analyzed separately in order to test the robustness of the approach in Section 5. Finally, we predict the amplitude and date of the current Solar Cycle 25 maximum and give our conclusions.

\section{A “Quasi”-Mechanism}

Stefani, Gieseke, and Weier (2019) cite the many authors who discussed whether the Hale ( $22 \mathrm{yr}$ ) cycle was synchronized by the alignment cycle of the tidally dominant planets Venus, Earth, and Jupiter. These authors acknowledged that " $a$ physically realistic synchronization mechanism based on these tides is still hardly conceivable". But they were able to build a dynamo model in which a kink-type Taylor instability led to oscillations of helicity and hence of the $\alpha$-effect related to it. Stefani, Gieseke, and Weier (2019) point out that "much current work focuses on a mechanism of soft modulation of solar activity: any such planetary influence could have enormous consequences for the predictability not only of the solar dynamo but, possibly, of the terrestrial climate, too". But in order to validate a mechanism for such planetary modulation, one must of course have strong observations to support it. Even more recently, Stefani et al. (2020) lend further support to such a mechanism and encouragement to pursue investigations: "While the long phase-coherent period in the early Holocene, together with the detailed analysis of the Schwabe cycle during the last 600 years, has lent greater plausibility to our starting hypothesis of a tidally synchronized solar dynamo, we would like to encourage more investigations into the Schwabe cycle during other periods".

We have recently detected oscillations with similar (pseudo-) periodicities in the SSA components of solar (Le Mouël, Lopes, and Courtillot, 2020a) and geophysical (Courtillot et al., 2013; Lopes, Le Mouël, and Gibert, 2017; Le Mouël, Lopes, and Courtillot, 2019a, 2019b, 2020b; Le Mouël et al., 2019) phenomena. We now believe that this is due to forcing (excitation functions) by the planets (see Malburet, ${ }^{1}$ 2019, for one of the early such types of analysis), due to their enormous orbital moments (the Jovian planets carry more than $99.8 \%$ of the total orbital moment of the solar system, $87 \%$ of this being due to Jupiter and Saturn alone). Jupiter has an orbital revolution period of $11.8 \mathrm{yr}$, that many authors have noted to be close to the mean Schwabe cycle of $11.2 \pm 3 \mathrm{yr}$ (it actually varies from 9 to $13 \mathrm{yr}$ ). This lack of perfect periodicity of the cycle lends itself particularly well to SSA and the decomposition in components it leads to. On the other hand, this lack of periodicity

\footnotetext{
${ }^{1}$ In 1918, then on the war front, Jean Malburet sent a sealed letter («pli secret ») to the Paris Academy of Sciences on links between the 11-year solar cycle and the ephemeris of the planets, to be opened 100 years after being submitted. The first author was asked by the Academy to review the letter and found it a remarkable early study of the subject that deserves being placed back at its historical place. Malburet (2019) leads to a handwritten facsimile, a typed version and an analysis of that remarkable paper.
} 
Table 1 The column labeled Associated periods lists SSA periods of planetary revolutions, their harmonics, and commensurable periods (from Mörth and Schlamminger, 1979; Le Mouël, Lopes, and Courtillot, 2020a). The column labeled SSN pseudo cycles lists SSA periods of SSN (from Le Mouël, Lopes, and Courtillot, 2020a). Periods are presented so as to show the association for each component. In most computations in this article the longer periods involving Neptune (in bold) are neglected because they are not short enough compared to the data length.

\begin{tabular}{|c|c|c|c|}
\hline \multicolumn{2}{|l|}{ Planet } & Associated periods $(\mathrm{yr})$ & SSN pseudo cycles \\
\hline \multicolumn{2}{|l|}{ Jupiter } & $\begin{array}{l}11.85 \text { (Schwabe) } \\
5.92\end{array}$ & $\begin{array}{l}10.56,11.3,13.43 \\
5.30,5.52\end{array}$ \\
\hline \multicolumn{2}{|l|}{ Saturn } & $31.44,15.72$ & $35.56,15.31$ \\
\hline \multicolumn{2}{|l|}{ Uranus } & $\begin{array}{l}83.1 \text { (Glcissbcrg) } \\
41.98\end{array}$ & $\begin{array}{l}90.03 \\
45.21\end{array}$ \\
\hline \multicolumn{2}{|l|}{ Neptune } & $\begin{array}{l}164.78 \text { (Jose) } \\
83.39\end{array}$ & $\begin{array}{l}\text { 131.02, 190.25 } \\
90.03\end{array}$ \\
\hline \multirow{2}{*}{$3\{$ Jupiter } & Saturn & $9.79,21.64$ (Hale) & $9.98,21.42$ \\
\hline & Uranus & $36.06,47.91$ & $35.56,45.21$ \\
\hline $4\{$ Uranus & Neptune & $40.40, \mathbf{1 2 4 . 3 7}$ & $45.21, \mathbf{1 3 1 . 0 2}$ \\
\hline 3 & 4 & $\begin{array}{l}15.30,25.09 \\
57.29,67.08 \\
9.38,31.02 \\
51.36,73.00\end{array}$ & $\begin{array}{l}15.31 \\
62.11 \\
9.98,35.56\end{array}$ \\
\hline
\end{tabular}

has led Charbonneau (2013) to abandon the idea of a planetary forcing. In contrast, we propose to follow a fully planetary course. For this, we use a method that draws on signal processing (time series analysis). We derive a filter, i.e. a transfer function that transforms the full spectrum of the ephemeris into the full spectrum of sunspots (that is both amplitudes and phases). This is legitimate since the planetary ephemeris (that are data) are sinusoids at the time scales we work with. The effects of the motions of the solar system planets are determined as components of a singular spectral analysis (SSA).

The revolution periods of the Jovian planets (Jupiter, Saturn, Uranus, and Neptune) are, respectively, $11.8 \mathrm{yr}, 31.4 \mathrm{yr}, 84.0 \mathrm{yr}$, and $164.8 \mathrm{yr}$ (Table 1). The closeness of Jupiter's revolution period and the Schwabe solar cycle has been noted since Wolf (1852). In our recent work, we have detected and/or confirmed the existence of $\approx 30$ and $\approx 60 \mathrm{yr}$ cycles in several geophysical series (Courtillot et al., 2013; Le Mouël, Lopes, and Courtillot, 2019a, 2019b, 2020b; Le Mouël et al., 2019) and also in sunspots (Le Mouël, Lopes, and Courtillot, 2020a). These can be linked to Saturn's rotation period (as already noted by many authors, e.g. Scafetta, 2020). The Gleissberg cycle (Gleissberg, 1939) at $\approx 90 \mathrm{yr}$ has been discussed for long and is very close to Uranus's rotation period (Le Mouël, Lopes, and Courtillot, 2017). It is more difficult to identify a signature of Neptune, since we have only 265 years of solar data versus the period of Neptune, $165 \mathrm{yr}$. However, Scafetta (2020) has noted a Jose cycle (Jose, 1965) around 155 to $185 \mathrm{yr}$. Given the oscillation of the orbit of each planet with respect to the ecliptic plane, one can expect to encounter oscillations with half periods, that is $5.5 \mathrm{yr}, 15 \mathrm{yr}, 42 \mathrm{yr}$, and $82 \mathrm{yr}$. Other harmonics of the Schwabe cycle have been identified (Courtillot and Le Mouël, 1976a, 1976b; Le Mouël, Lopes, and Courtillot, 2019a, 2020a; see the review in Petrovay, 2020).

We derive the transfer (or excitation) function for Jupiter in Section 3. But we can consider a larger number of transfer or excitation functions as shown in Section 4. In order not to burden the main body of the paper, this is further discussed in Appendix A. 
Now, one can explore beyond the set of orbital periods of the Jovian planets. We bypass the impossibility to solve the N-body problem for $\mathrm{N}>2$ by using the concept of commensurability (that is when the ratio of the periods of two planets can be expressed as a fraction with integer numerator and denominator less than 9 - Mörth and Schlamminger, 1979; Okhlopkov, 2016; Scafetta, 2020). In this way, planets encounter a resonance and can be paired, and each pair considered as a single object. In our case, Jupiter/Saturn and Uranus/Neptune form two pairs. Pairs of pairs can also be considered, thus the set (Jupiter/ Saturn)/(Uranus/Neptune).

The SSA periods common to the Jovian planets of the solar system (periods of planetary revolutions and commensurable periods) and to SSN are given in Table 1 (from Mörth and Schlamminger, 1979; Le Mouël, Lopes, and Courtillot, 2020a).

\section{Transfer Functions: From Planetary Aphelia to Sunspot Numbers}

First, we have analyzed the SSN series using singular spectrum analysis (SSA) as in Le Mouël, Lopes, and Courtillot (2017). We use monthly values in all computations (all have been performed with the highest possible resolution of the data ${ }^{2}$ ). Figure $2 \mathrm{a}$ represents the first three SSA components of SSN, i.e. a trend, an $\approx 11 \mathrm{yr}$ component (Schwabe) and an $\approx 84 \mathrm{yr}$ component (Gleissberg). These components carry, respectively, 24\%, 26\% and 9\% of the total variance. Figure $2 \mathrm{~b}$ shows the reconstruction of the series using only these first three SSA components: they capture 59\% of the total variance. Note that the second (Schwabe) component is not periodical but quasi-periodical as is the case for the observational data (sunspot cycles). The reconstruction can be improved by using components of higher order, notably corresponding to the half-periods of the Jovian planets (Appendix A and Section 4).

Once the periodicities have been extracted from SSN using SSA, we compare them with the various periodicities in the ephemeris of the planets (Table 1, Appendix B). Although we of course acknowledge that we do not grasp fully the physics of the interactions and forcings of the planets on the solar photosphere, we assume that there exists a linear filter that allows one to pass from the planetary aphelion to the solar cycles. We calculate this transfer function (both modulus and phase) using a Hilbert transform (see Papoulis, 1977). Let us apply this line of reasoning to the couple made of the aphelion of Jupiter and the Schwabe solar cycle. Figure 3 shows the $\approx 11 \mathrm{yr}$ aphelion of Jupiter (in black) and the corresponding SSA component of the sunspot number (in red), and in blue the small and quasi-regular phase drift $\phi_{11}(t)$ (bottom) and significant modulation $\alpha_{11}(t)$ (top) of the transfer function (recall that, for a planet, aphelia times sine of declination is the gravity potential, up to a constant, and, with another constant, gives the moment of inertia). As a check, we apply this transfer function to the aphelion of Jupiter $\rho_{J u p}$ :

$$
\operatorname{SSN}_{11}=\alpha_{11}(t) \times \rho_{\text {Jup }}\left(t+\phi_{11}(t)\right)=\alpha(t)_{11} \times \sin \left(\omega_{\text {Jup }} t+\phi_{11}(t)\right)
$$

We get the result shown in Figure 4. The curve with black dots is the aphelion of Jupiter convolved with the transfer function that has the phase and amplitude of the Schwabe cycle (see Figure 2a middle row and Figure 3), in red is the Schwabe SSA component of SSN. The two are so close that the black curve is almost indistinguishable from the red one: the task of

\footnotetext{
${ }^{2}$ There are actually many available sunspot series, given on daily, monthly, annual (12 month), and 13-month time scales. For reasons that are not easily understood, there are slight differences that lead to slightly different spectra. For instance, the peak value of the Schwabe cycle can range from 10.5 to 11.2 years. We have selected the Royal Observatory of Belgium data at monthly intervals.
} 

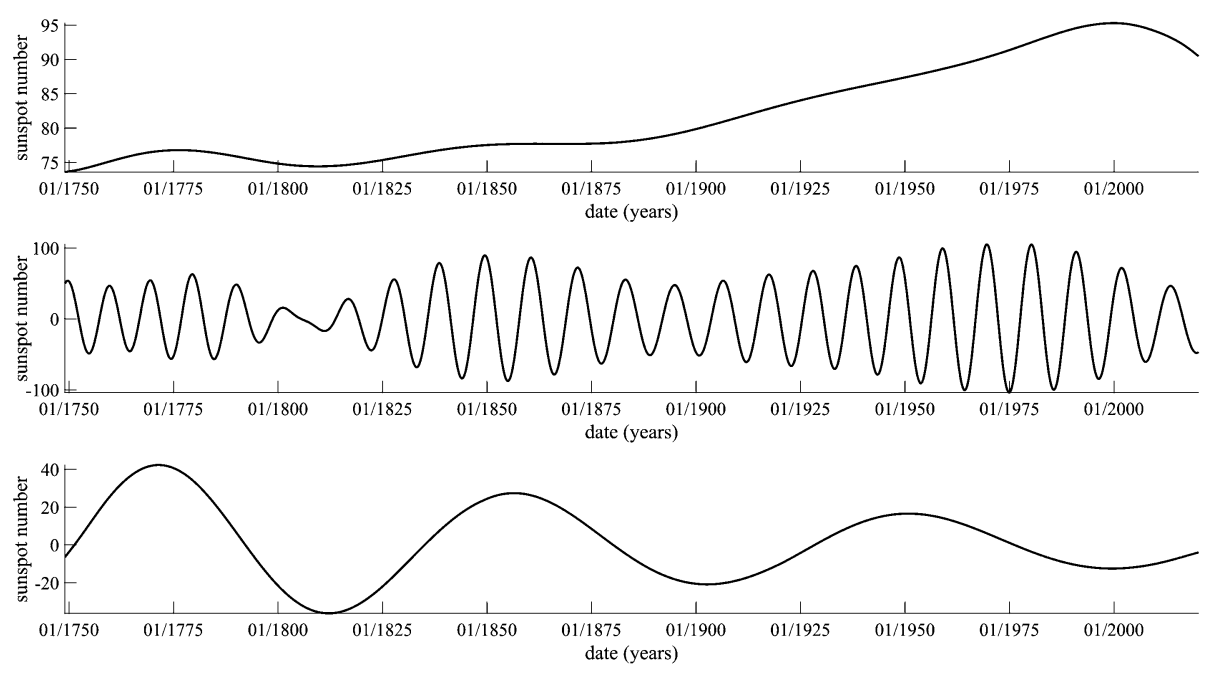

(a)

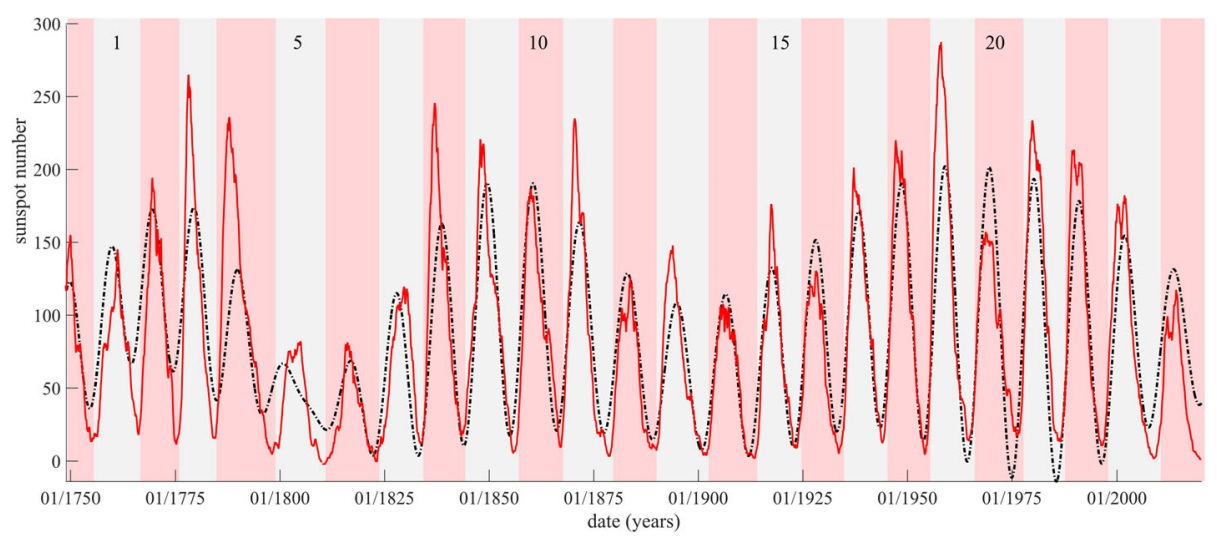

(b)

Figure 2 (a) The first three components of the SSA analysis of SSN: trend, Schwabe and Gleissberg. (b) Comparison of the sum of the first three SSA components of SSN (black) with the annual mean data (in red).

finding a transfer function is fully successful. We choose to model the "transformed" Jupiter aphelion (that is the transfer function applied to the aphelion) by a sum of sine functions. We search for the minimum number $N$ of sine functions to be used (given a certain precision level), using simulated annealing (Kirkpatrick, Gelatt, and Vecchi, 1983). The result has the form

$$
\Sigma \alpha_{k} \times \sin \left(\omega_{k} t\right) \quad(k=1, \ldots, N) .
$$

This is a rather simple inverse problem, in which one must find the value of parameters $\alpha(t)$ and $\omega(t)$ and their number $N$. An infinite sum would (over) explain the data. Here, we try to account for the data with the smallest possible number of sine functions (in the sense of the $\chi^{2}$ statistics). $N$ is this number. Once two models have been found, one with $\mathrm{k}$ sinuses and the other with $\mathrm{k}+1$, a statistical independence test between the data and the two models is performed. If model $\mathrm{k}+1$ does not explain the data better than model $\mathrm{k}$, we keep model $\mathrm{k}$. 


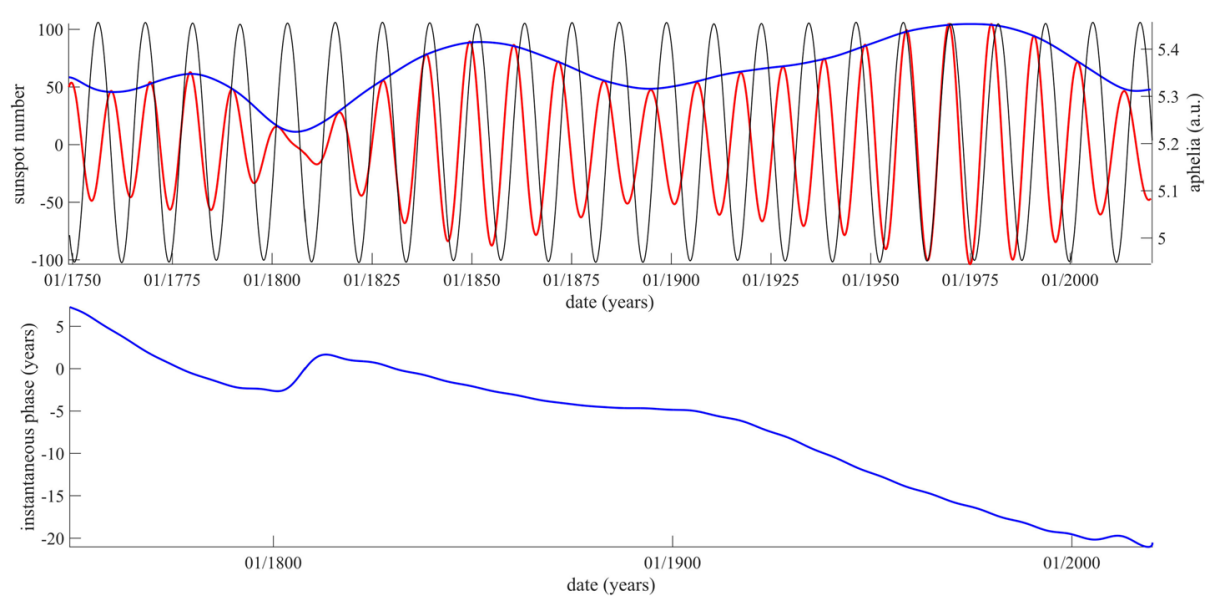

Figure 3 In black the aphelion of Jupiter, in red the Schwabe SSA component of the sunspot series (i.e. the solar cycle). In blue the modulus (top) and phase (bottom) of the transfer function between the two.

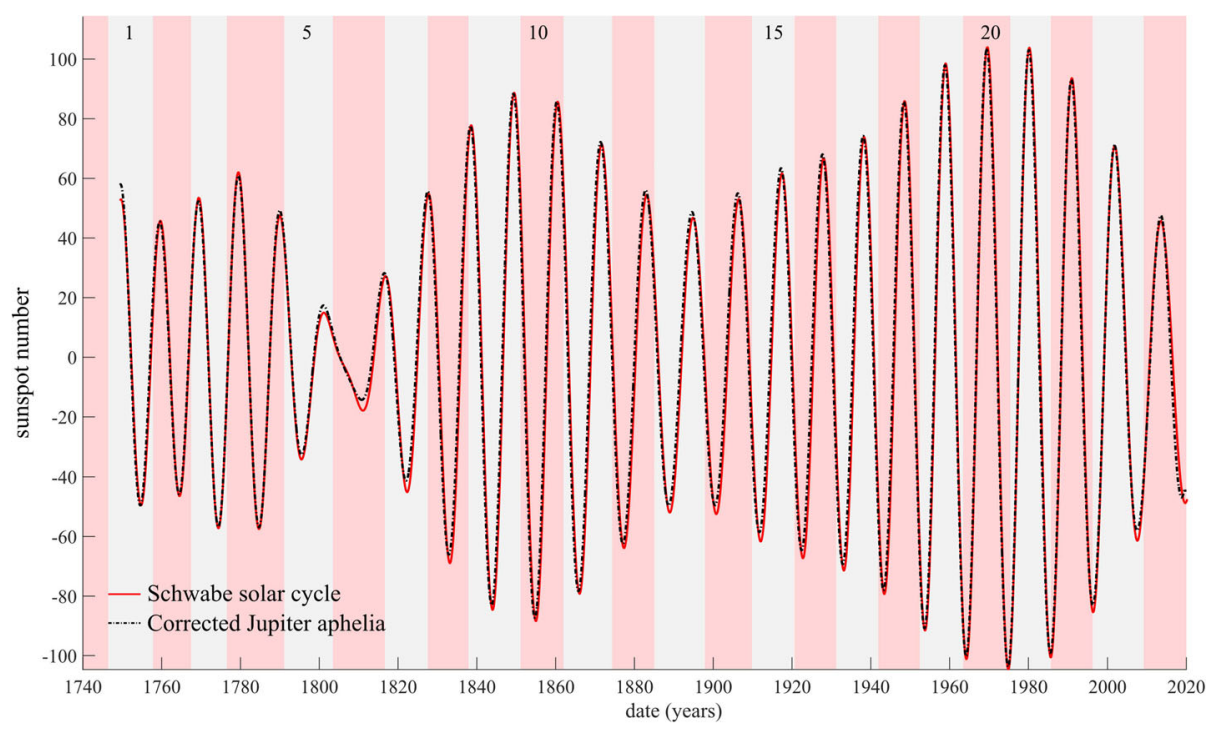

Figure 4 In black the aphelion of Jupiter transformed using the phase and amplitude of the Schwabe cycle (see Figure 2a middle row and Figure 3), in red the Schwabe SSA component of SSN. The two are so close that the black curve is almost hidden behind the red one.

Otherwise, we iterate the inversion process once more (see Courtillot et al., 2013 for more details).

In order to reconstruct the Schwabe SSN component of SSA (Figure 4), five sine functions are necessary and sufficient $(N=5)$. Two of the $\omega_{k}$ sine functions correspond to $11 \mathrm{yr}$ and $90 \mathrm{yr}$, that is the Schwabe and Gleissberg cycles, respectively, linked to Jupiter (Table 1, 11.85 vs 11.3) and Uranus (Table 1, 83.97 vs 90.03). But, as we can see in Figure 3, these are pseudo-periodical and the three remaining sine terms allow a better overall fit (on the order of 99.9\%), for instance of the anomaly between Solar Cycles 5 and 6 (Figure 5). 


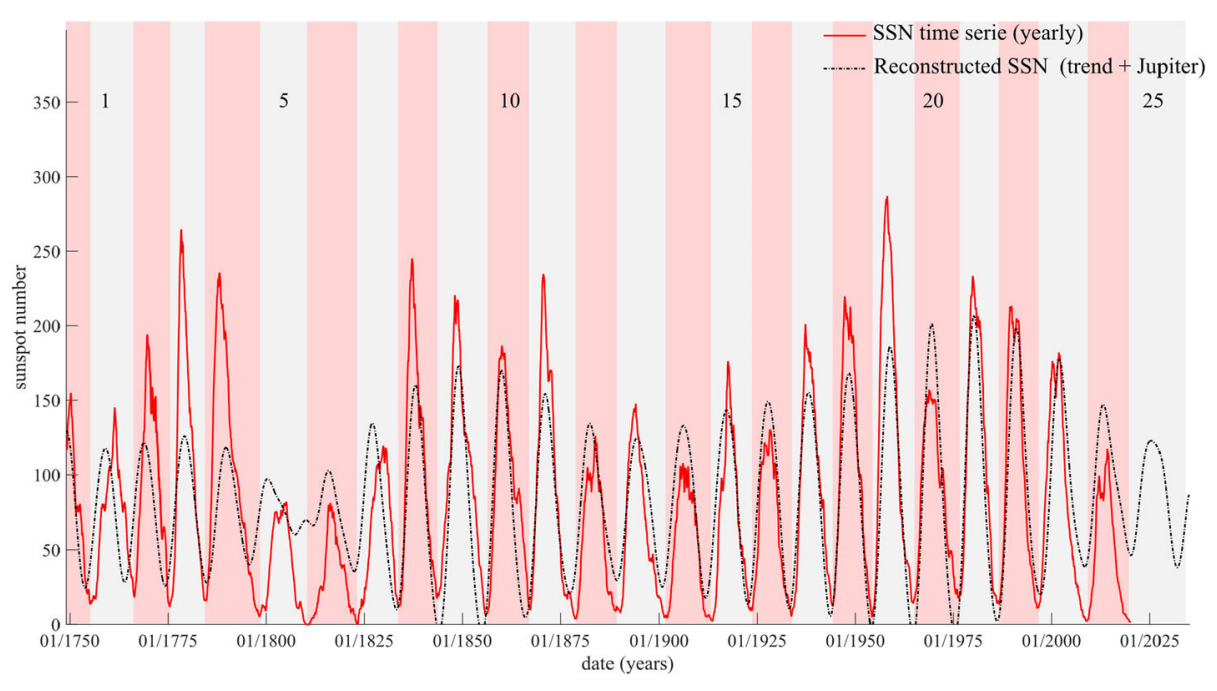

Figure 5 In red the full SSN as in Figure 1, in black the theoretical reconstruction (with SSA trend added), both from 1749 to 2020 . The theoretical curve has (arbitrarily) been continued to 2035 (a 15 year prediction).

For completeness, we give the derivation of the equivalent of Figures 3 and 4 for components linked to Uranus (Gleissberg) and Saturn (30 yr) in Appendix A. For all the commensurable periods, the ephemerids precede the solar response. The transfer functions (filters) for all components have slow, smooth variations, and phases do not jump: this implies that the filters are invariable by time translations; hence, they can have a causal, physical meaning (Papoulis, 1977): we conclude that planetary variations are likely to be the cause of the solar variations by some physical process.

With such a model (transfer function), we can forecast the sunspot series in the future, for instance until January 1, 2035 (Figure 5). For this, we simply continue the five sine functions obtained above, including the first (90 yr) component that plays the role of a trend. The fit is not bad but it has some small problems, for instance around 1810 . We now attempt to improve both the fit and the prediction.

\section{Reconstructing Past and Predicting Future Solar Activity}

\subsection{Retaining Only Jupiter (First Order)}

In the previous section we have outlined our method to reconstruct the potential influence of Jupiter on solar activity, the former being characterized by its ephemeris and the latter by the SSN series. We have thus constructed a filter (transfer function) to transform the former into the latter (Section 3). The transfer function can be used to predict to first order the continuation of the SSN series (for example to 2035 in Figure 5). The reconstruction of SSN encounters small misfits in Solar Cycles 2 to 6, whose amplitude is underestimated (amplitude of the black curve smaller than that of the red curve in Figure 5). There is a significant discrepancy in time around 1810 ( \pm 10 years). The predicted Solar Cycle 25, has a mean peak amplitude of 121.4 in 2025.3. But we expect to do better by taking into account 


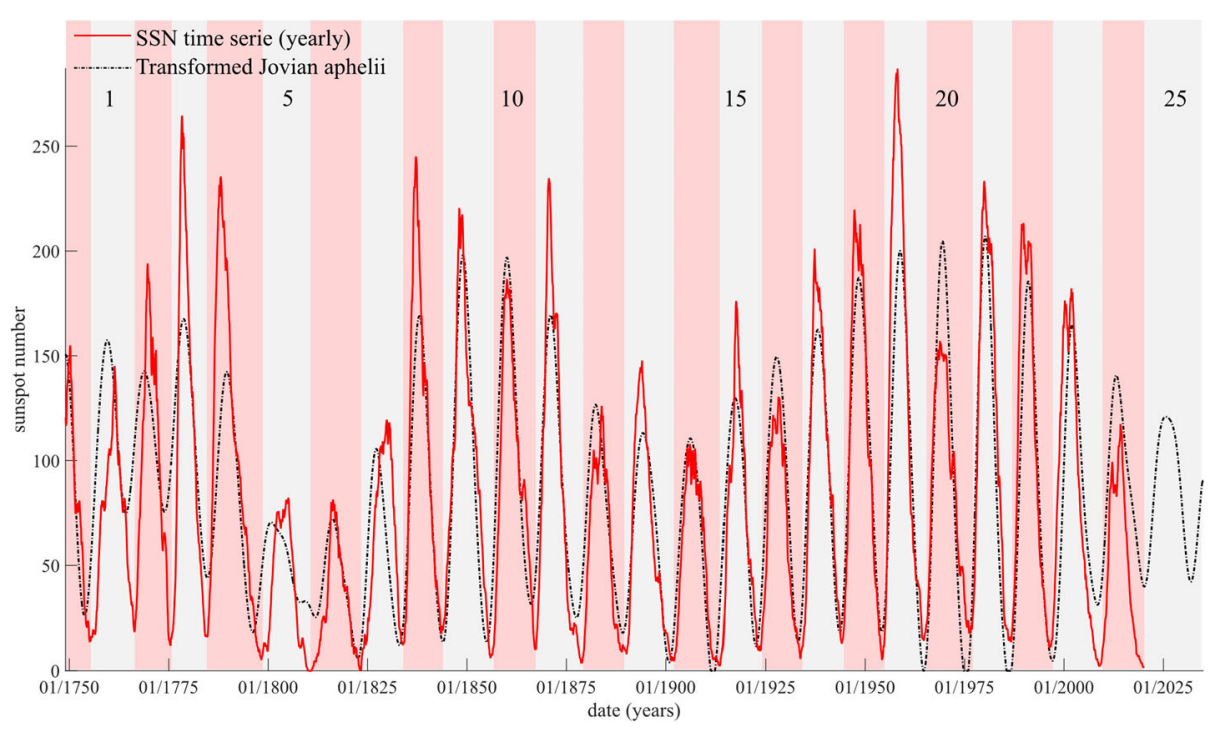

Figure 6 In red, the yearly SSN time series. In black the reconstructed series, taking into account the ephemeris of all four Jovian planets, extended to 2035, thus a possible prediction of Solar Cycle 25.

the influence of the other Jovian planets. ${ }^{3}$ Altogether, with five sine terms, the variance accounted for is $85.8 \%$, and the root mean square error between reconstructed and observed series is $12.4 \%$.

\subsection{With the Jovian Planets but Without Their Interactions (Second Order)}

The SSA analysis of SSN yields a number of (pseudo-)periodic components that are close within the uncertainty to those encountered in the aphelia of the Jovian planets (Table 1). In the same way that components at 11 and $5.5 \mathrm{yr}$ were associated with Jupiter, $90 \mathrm{yr}$ can be linked to Uranus, and 30 and 15 yr to Saturn. Applying the method outlined in Section 3, we obtain Figure 6. Here again, five sine functions are necessary and sufficient $(N=5)$. The variance accounted for is $91.4 \%$, and the root mean square error between reconstructed and observed series is $9.1 \%$. Despite an overall good fit to the observed SSN, a number of misfits remains: phase and amplitude offsets in Solar Cycles 1 to 6, a major misfit around 1800, a general underestimation of cycle amplitudes in the 20th century, a very different envelope of maxima of Solar Cycles 17 to 21, and an overestimated minimum before Solar Cycle 24. Should we stop the analysis at this point, the «predicted» Solar Cycle 25 would peak at 119.4 in 2025.0 .

\subsection{With the Jovian Planets and Their Interactions (Third Order)}

We go one step beyond by taking into account the interactions between pairs and pairs of pairs of commensurable planets (Jupiter/Saturn, Uranus/Neptune and (Jupiter/Saturn)/ (Uranus/Neptune)) as explained in Section 2. The result is shown in Figure 7. Here, 23 sine

\footnotetext{
${ }^{3}$ Because this is an incomplete reconstruction, values are sometimes slightly negative due to the decomposition in the sum of a trend and zero mean oscillatory terms (Figure 5 near 1850, 1950 to 1975).
} 


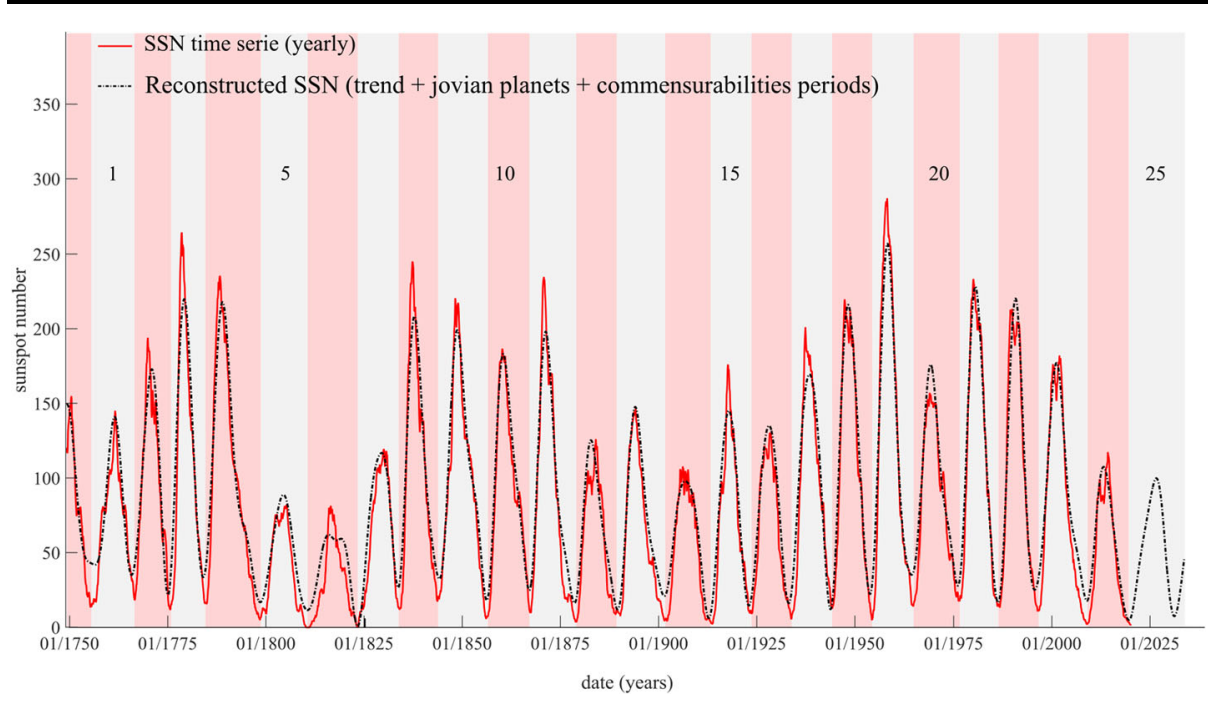

Figure 7 In red the series of annual means of SSN. In black the sum of SSA components with periods compatible with the revolution periods of Jovian planets and the periodicities of the ephemeris of commensurable pairs Jupiter/Saturn and Uranus/Neptune and pair of pairs (Jupiter/Saturn)/(Uranus/Neptune) (see Table 1).

functions are necessary and sufficient $(N=23$, that is the 22 periods listed in Table 1 plus one to fit the trend). The variance now accounted for is $95.6 \%$, and the root mean square error between reconstructed and observed series is 5.2\%. Many of the remaining shortcomings seen in Figure 6 are satisfactorily corrected. The fits of the theoretical SSN curve to most cycle maxima and minima are very good. The fit to Solar Cycles 1 to 6 and, in particular, to the two very small Solar Cycles 5 and 6 is very good, except for the first minimum which is underestimated. The pattern of maxima of Solar Cycles 16 to 23 and, in particular, the small Solar Cycle 20 are correctly reconstructed. The new «theoretical» SSN curve (in black on Figure 7) can be extrapolated, providing a new, we hope improved, prediction of Solar Cycle 25 date (2026.2) and amplitude (97.6) of maximum. Recall that we have performed all computations with monthly means.

\section{Discussion}

Let us come back to the three reconstructions of SSN based on an increasing number of planetary aphelia: Figure 5 involves Jupiter alone, Figure 6 the four Jovian planets, and Figure 7 has the addition of two interacting pairs (Jupiter/Saturn and Uranus/Neptune) and one pair of pairs (Jupiter/Saturn)/(Uranus/Neptune) as indicated in Section 3. In these three figures, the sunspot data SSN are in red and the reconstructions in black (dotted). We can see that the reconstructions improve as planets then planet pairs are added. For instance, Solar Cycles 5 and 6 (1800-1825) are much better reconstructed in Figure 6 than 5, although the fit is still imperfect. On the other hand, the two minima surrounding Solar Cycle 24 (2005-2015) remain much too high and fail to "touch down" near zero. Figure 7 shows very significant improvements in all instances. The reconstructions of Solar Cycles 5 and 6, and that of Solar Cycle 24, are quite good. We could possibly still improve the reconstruction marginally by introducing the terrestrial planets but this is not useful given the quality of the fit in Figure 7. 
It is not so straightforward to determine uncertainties in reconstructions and predictions. The ephemeris for instance are given without uncertainties (they are supposed to be known exactly). In Table 2, we list the observed (in red) vs. reconstructed (with our method - in black) dates and amplitudes of solar cycle maxima. The date misfit ranges from 1.1 year in advance to 1.2 year late ( -0.30 and 0.68 year for the median and standard deviation); the maximum value misfit ranges from -44.7 to 7.8 sunspots ( -8.95 and 15.7 sunspots for the median and standard deviation).

We can further test the accuracy of the method by attempting a number of hindcasts. That is, we have used the SSN data from 1749 to successively 1976, 1997, and 2010 and performed a reconstruction using the planetary aphelia as explained in this article (Sections 2 and 3; Figure 8). We have then used this reconstruction to predict the following cycle, respectively 21 (Figure 8a), 22 (not shown to save space), 23 (Figure 8b), and 24 (Figure 8c).

We see that the predictions are quite good: the root mean square (RMS) of the difference between predicted and observed SSN values in a solar cycle equals 10.4 sunspots $(4.5 \%$ of the maximum), $-1.6 \%$ misfit of the maximum, and 6 months misfit of its date for Solar Cycle 21, respectively; 17.0 sunspots ( $7.7 \%$ of the maximum), $3.6 \%$ of the maximum, 11 months for Solar Cycle 22; 10.7 sunspots (6.1\% of the maximum), $-3.8 \%$ of the maximum, -8 months for Solar Cycle 23; and 10.2 sunspots (10.1\% of the maximum), $-13.6 \%$ of the maximum, -12 months for Solar Cycle 24. The maximum of Solar Cycle 24 was 117.4 in 2014.2. This is to be compared with our prediction of 101.5 in 2013.2.

The RMS and standard deviations of differences between hindcast (i.e. "post-predicted") and observed SSN values in Solar Cycles 21, 22, 23, and 24 (for the full cycle at monthly sampling rate) are, respectively, (in sunspot units) -1.0 and 10.4, 4.3 and 16.6, -3.5 and 10.1, and 3.2 and 9.8. These statistical estimates give us some confidence in the results of reconstructions and hindcasts, therefore, in our SSA based forecast (Figure 8 and Table 2). This can be used to estimate roughly the uncertainties in future predictions, such as for Solar Cycle 25 (see below): under 10\% for the amplitude and 1 year for the date.

In the hindcasts, a significant part of the data are used in all cases, hence the predictions are not independent. Another test we have performed consists in having broken the original time series in two almost equal halves (ensuring independence) that were analyzed in the same way and compared (the separation occurs in 1900 at the end of Solar Cycle 13). The signal not being strictly stationary, the Hankel matrices are expected to be different, the eigenvalues and eigen vectors also. In Figure 9a, we show the first three SSA components of the first half of the data and the first two components of the second half. All these components correspond to purely Jovian periods.

In this paragraph, we comment on the notion of trend. In most reconstructions, we call the first SSA component the trend (f.i. Figure 2a, upper curve). Actually, this trend could be a segment of a component with a longer period, similar to or longer than, say, half of the data interval. This is the reason why the trend could correspond to some of the components of the ephemeris of Neptune (in bold in Table 1). This trend is added to Jupiter's effect (Figure 5), Jupiter+Uranus+Saturn (Figure 6), and Jupiter+Uranus+Saturn+ Jupiter/Saturn pair (Figure 7). For more on that approach to trends; see Rekapalli and Tiwari's (2020) article. Rekapalli and Tiwari (2020) showed the presence of a Jose solar cycle $(\approx 160 \mathrm{yr})$ in a $400 \mathrm{yr}$ long series of total solar irradiance, that is the revolution period of Neptune.

In Figure 9 (left) the first slowly varying component (in blue) could be called the trend or correspond to a weak $\approx 90 \mathrm{yr}$ oscillation. The second component (in red) has a (pseudo-)period of 64 years. This cycle results from a combination from Uranus (84 yr) and Saturn (30 yr) (Scafetta, 2020; Le Mouël, Lopes, and Courtillot, 2020a). If we combine components 1 and 2 from the first half of the data, the resulting oscillation connects nicely 


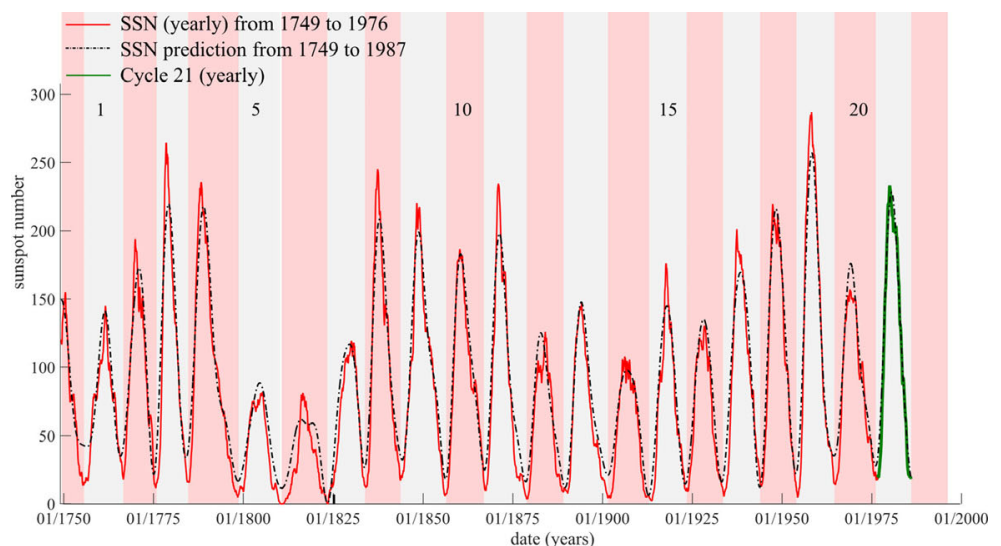

(a)

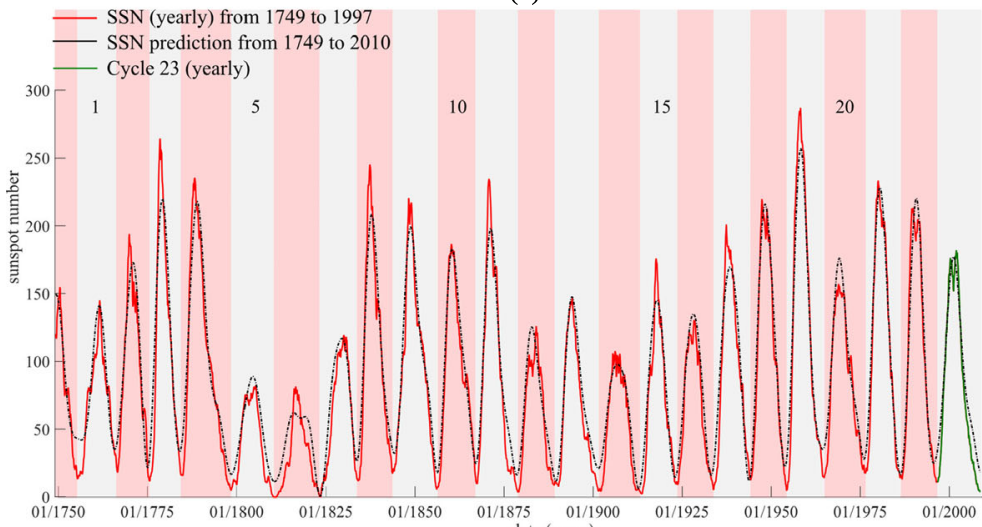

(b)

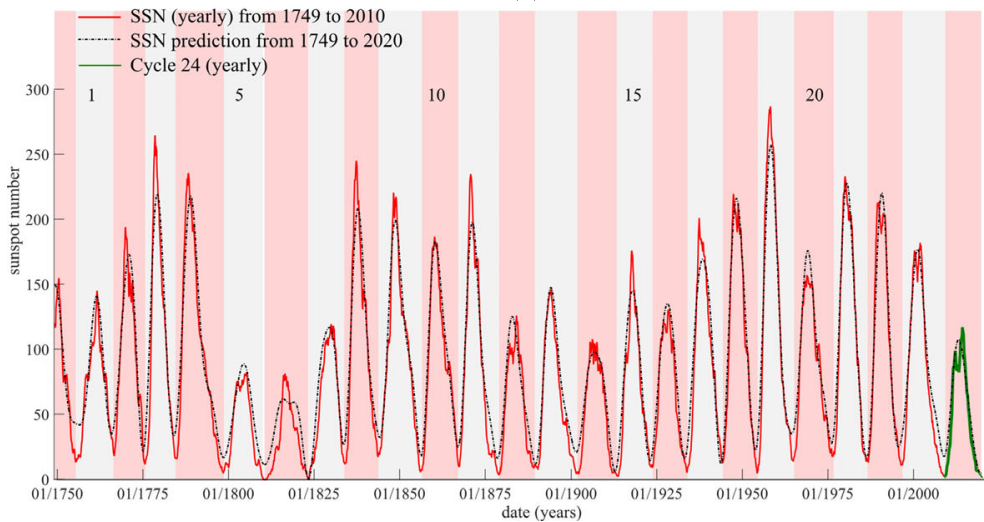

(c)

Figure 8 (a) "Prediction" of Solar Cycle 21. In red SSN annual mean from 1749 to 1976, in green Solar Cycle 21 (annual data), in black the reconstructed SSN curve using as input data the red values and thus predicting SSN from 1976 to 1987. (b) "Prediction" of Solar Cycle 23. In red SSN annual mean from 1749 to 1997, in green Solar Cycle 23 (annual data), in black the reconstructed SSN curve using as input data the red values and thus predicting SSN from 1997 to 2010. (c) "Prediction" of Solar Cycle 24. In red SSN annual mean from 1749 to 2010, in green Solar Cycle 24 (annual data), in black the reconstructed SSN curve using as input data the red values and thus predicting SSN from 2010 to 2020. 
Table 2 First three columns: dates of cycle maxima, in red observed, in black reconstructed in this article, difference in blue. Last three columns values of cycle maxima, same color code.

\begin{tabular}{|c|c|c|c|c|c|}
\hline \multicolumn{2}{|c|}{ Maxima Dates } & \multirow{2}{*}{$\frac{\text { year }}{0.2}$} & \multicolumn{2}{|c|}{ Sunspot Numbers } & \multirow{2}{*}{$\frac{\%}{5.5}$} \\
\hline $15 / 06 / 1761$ & $15 / 04 / 1761$ & & 145.2 & 137.2 & \\
\hline $15 / 10 / 1769$ & $15 / 10 / 1770$ & -1.0 & 194.1 & 170.7 & 12.1 \\
\hline $15 / 06 / 1778$ & $15 / 01 / 1779$ & -0.6 & 264.6 & 219.9 & 16.9 \\
\hline $15 / 02 / 1788$ & $15 / 11 / 1788$ & -0.8 & 235.4 & 219.1 & 6.9 \\
\hline $15 / 02 / 1805$ & $15 / 05 / 1804$ & 0.8 & 82.0 & 81.6 & 0.5 \\
\hline $15 / 06 / 1816$ & $15 / 11 / 1815$ & 0.6 & 81.2 & 52.2 & 35.7 \\
\hline $15 / 11 / 1829$ & $15 / 05 / 1829$ & 0.5 & 119.3 & 111.6 & 6.5 \\
\hline $15 / 04 / 1837$ & $15 / 10 / 1837$ & -0.5 & 245.0 & 208.1 & 15.1 \\
\hline $15 / 02 / 1848$ & $15 / 08 / 1848$ & -0.5 & 220.5 & 198.5 & 10.0 \\
\hline $15 / 02 / 1860$ & $15 / 05 / 1860$ & -0.2 & 186.7 & 181.3 & 2.9 \\
\hline $15 / 09 / 1870$ & $15 / 03 / 1871$ & -0.5 & 234.7 & 197.7 & 15.8 \\
\hline $15 / 12 / 1883$ & $15 / 09 / 1882$ & 1.2 & 126.2 & 120.0 & 4.9 \\
\hline $15 / 02 / 1894$ & $15 / 01 / 1894$ & 0.1 & 147.8 & 143.6 & 2.8 \\
\hline $15 / 02 / 1906$ & $15 / 11 / 1906$ & -0.7 & 107.8 & 91.3 & 15.3 \\
\hline $15 / 08 / 1917$ & $15 / 11 / 1917$ & -0.3 & 176.1 & 141.5 & 19.6 \\
\hline $15 / 04 / 1928$ & $15 / 02 / 1928$ & 0.2 & 130.4 & 130.5 & -0.1 \\
\hline $15 / 05 / 1937$ & $15 / 06 / 1938$ & -1.1 & 201.1 & 167.8 & 16.6 \\
\hline $15 / 05 / 1947$ & $15 / 04 / 1948$ & -0.9 & 219.8 & 216.6 & 1.5 \\
\hline $15 / 03 / 1958$ & $15 / 04 / 1958$ & -0.1 & 287.0 & 260.1 & 9.4 \\
\hline $15 / 11 / 1968$ & $15 / 03 / 1969$ & -0.3 & 157.1 & 173.3 & -10.3 \\
\hline $15 / 01 / 1980$ & $15 / 07 / 1980$ & -0.5 & 233.4 & 229.7 & 1.6 \\
\hline 15/11/1989 & $15 / 10 / 1990$ & -0.9 & 213.3 & 221.1 & -3.7 \\
\hline $15 / 12 / 2001$ & $15 / 04 / 2001$ & 0.7 & 182.2 & 175.3 & 3.8 \\
\hline $15 / 04 / 2014$ & $15 / 03 / 2013$ & 1.1 & 117.4 & 107.5 & 8.4 \\
\hline
\end{tabular}

with the first component of the second half and the resulting curve is close to the envelope of the total signal (Figure 9b). When all these components are added and juxtaposed, the comparison between the original SSN series (from Figure 2b) and the curve reconstructed from a concatenation of the SSA components of the two halves of the series (Figure 10) is equally good.

In Figures 5 to 7, we have extended our reconstruction by 10 years, thus providing "predictions" of Solar Cycle 25. Only the prediction in Figure 7 seems acceptable, with its two surrounding minima reaching down almost to zero. Figure 11 shows a close-up, with monthly mean values on the left and annual on the right. The maximum amplitude predicted for Solar Cycle 25 is $97.6 \pm 7.8$ and its date $2026.2 \pm 1$ year.

Another test consists in seeing whether the SSA method applied to only half of the data retains the same ability to predict the following cycle. Using the SSA components shown in Figure 9, we attempt to predict Solar Cycle 14 from the 13 previous cycles, from 1 to 13 (Figure 12a). The predicted Solar Cycle 14 is quite good: the date of the maximum is January 1906 vs. the observed February 1906, the date of the next minimum is June 1912 vs. November 1911, the amplitude at the maximum is 131.2 vs the observed 107.8 , and the amplitude at the minimum is 15.6 vs. 6.2. The predicted duration of Solar Cycle 14 is 11.8 yr vs. the observed $10.4 \mathrm{yr}$. The date of the maximum is off by only 1 month and the duration of the cycle by $1.4 \mathrm{yr}$. The amplitude is off by $20 \%$. 


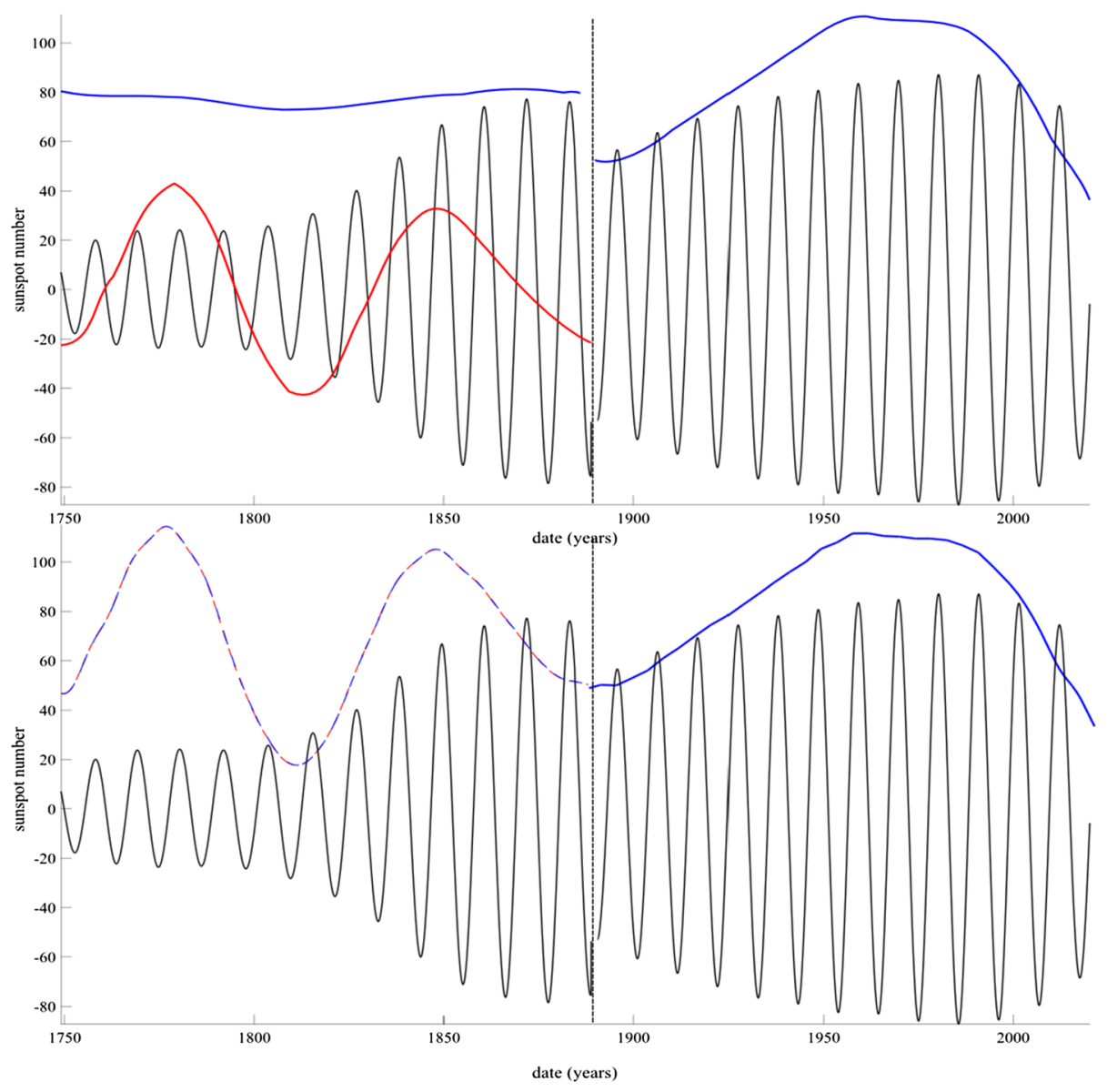

Figure 9 (top) First three (left) and two (right) SSA components for, respectively, the first half (1749-1889) and second half (1889-2020) of the SSN sunspot series, respectively. (bottom) Same but with components 1 and 2 of the first half combined.

We next try to predict Solar Cycle 25 from the 11 previous Solar Cycles 14 to 24 . In Figure $12 \mathrm{~b}$ we compare the Solar Cycle 25 predicted from only half of the available data to the one predicted from the full data set (Figures 8c and 11). The minimum of Solar Cycle 25 (at minus 53) when predicted with half the SSN data raises a problem, since SSN cannot be negative. This is due to the fit of the trend, that is component 1 , by a sine function. If the curvature of the trend is too strong on an interval too short to capture the true period of the associated planetary period, the extrapolation of that trend can be negative, that is physically impossible. We see that if the full data set is used in the SSA analysis, the trend becomes acceptable and the predicted Solar Cycle 25 (in green dots in Figure 12b) recovers physically plausible characteristics. The predicted date of the maximum from half of the data set is May 2023 vs. the "full" prediction which is March 2026, the date of the next minimum is October 2028 vs. February 2031, the amplitude at the maximum is 97.6 vs. 97.6, and the amplitude at the minimum is -53 vs. 3.1. The predicted duration of Solar Cycle 25 is $9.5 \mathrm{yr}$ with only half of the data vs. $11.7 \mathrm{yr}$ for the full data set. 


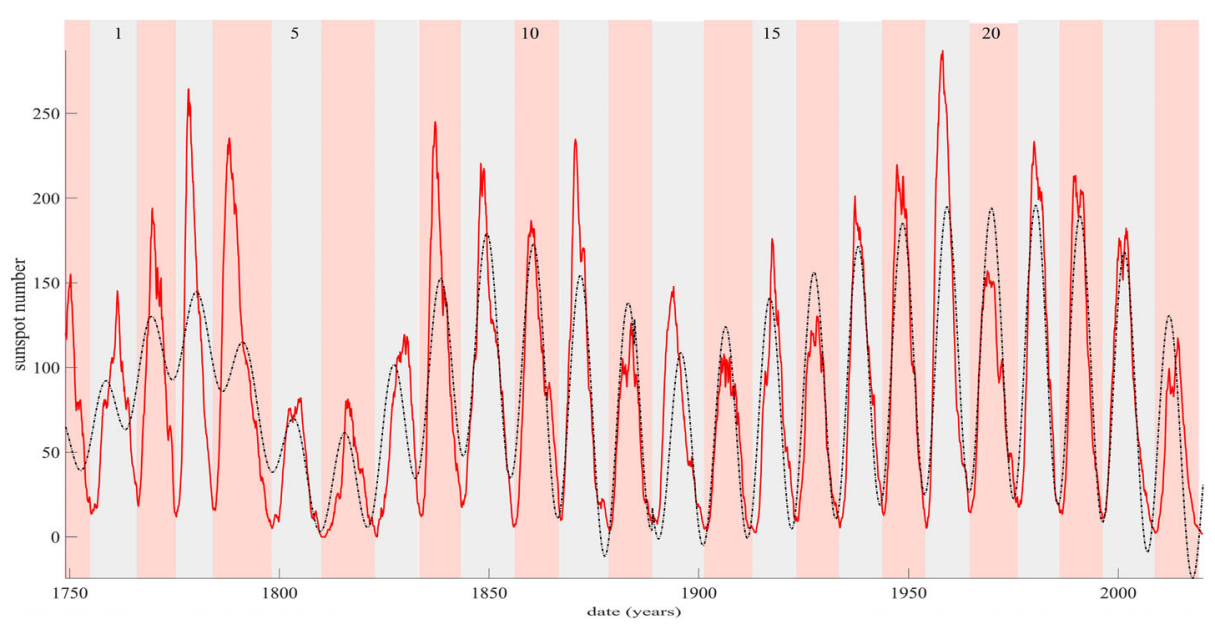

Figure 10 Comparison of the sum and concatenation of the eigen vectors of Figure 9 (in black) with the SSN time series (in red).

Figure 11 In red the observed series of SSN means (left monthly means, right annual means). In black the sum of SSA components with periods compatible with the revolution periods of the Jovian planets and the periods of the ephemeris of commensurable pairs

Jupiter/Saturn and

Uranus/Neptune and pair of pairs (Jupiter/Saturn)/(Uranus/

Neptune), extended to 2035.

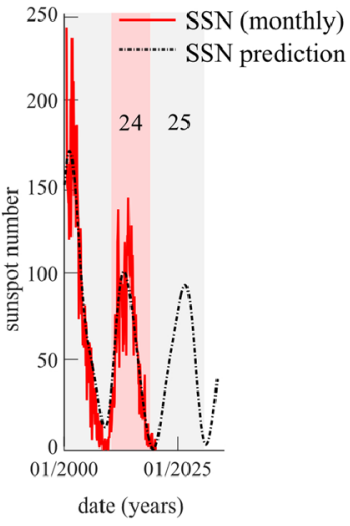

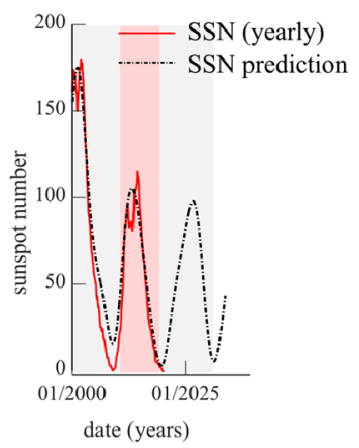

We can compare our prediction with the selection of some 15 early forecasts for Solar Cycle 25 tabled in Petrovay's (2020) review (Table 3). The predicted amplitudes of the maximum range from 57 \pm 17 (Covas, Peixinho, and Fernandes, 2019; neural network category) to 175 ( $\mathrm{Li}$, Feng, and $\mathrm{Li}, 2015$; internal precursor category) and the dates of the maximum from 2022 (Attia, Ismail, and Basurah, 2013; neural network category) to 2027.2 \pm 1.0 (Labonville, Charbonneau, and Lemerle, 2019; dynamo model category).

Our method belongs to the spectral category, and also to the model-based category, given that we do propose a (quasi-) physical model involving the planets. Compared to the distribution of early forecasts tabled by Petrovay (2020), our date for the maximum of Solar Cycle 25 is on the late side and our amplitude on the lower range. Solar Cycle 25 is predicted to be a small one, very similar to Solar Cycle 24 and similar to the Solar Cycles 5-7 (the Dalton minimum) and 12-16 (the Gleissberg minimum); as such the pair 24-25 (and 26?) may come to be known as "the Modern minimum". 


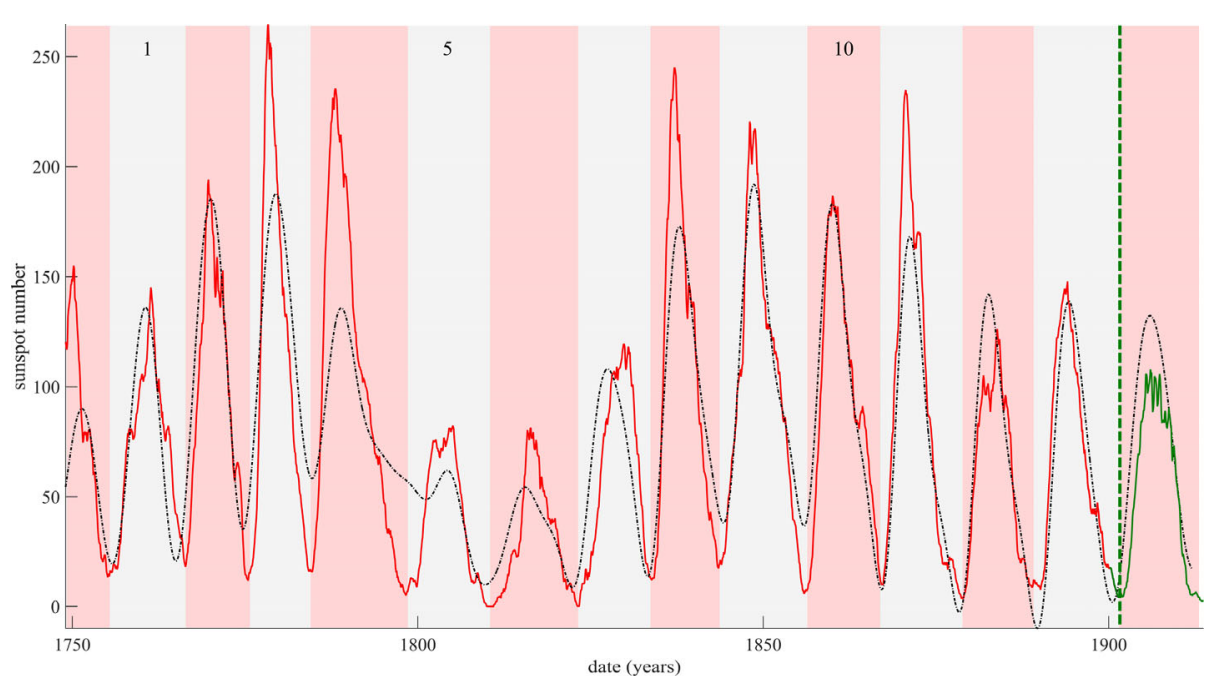

(a)

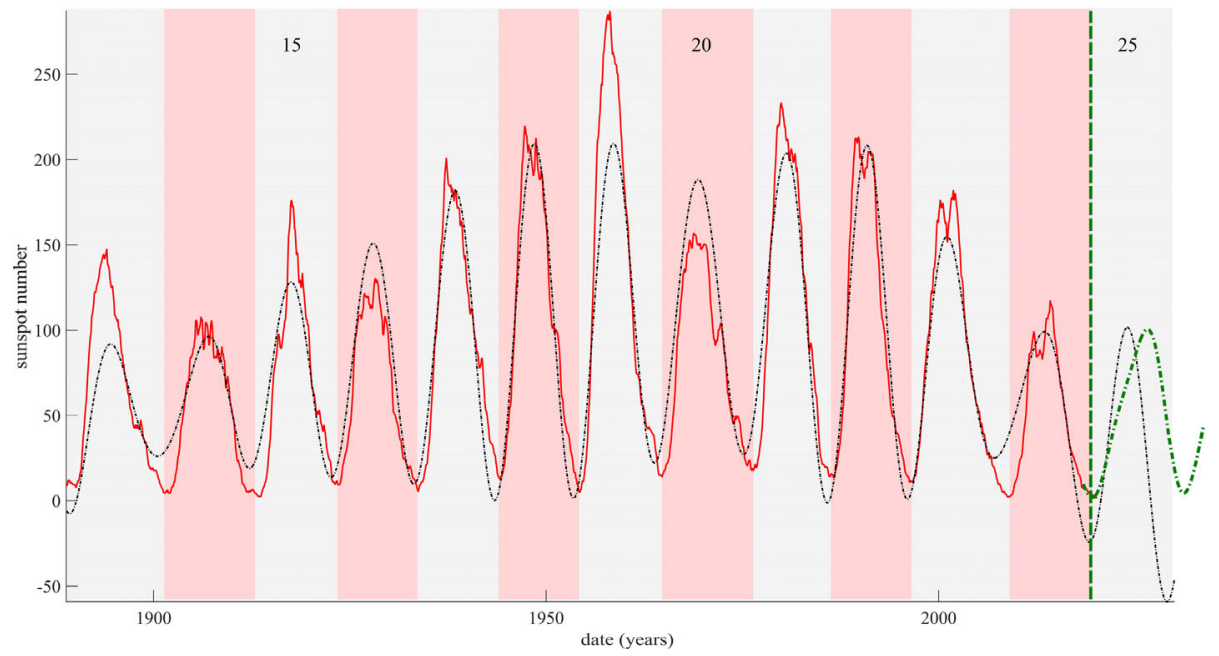

(b)

Figure 12 (a) In red SSN Solar Cycles 1 to 13 (observed), in dotted black the reconstruction using SSA Solar Cycles 1 to 13 as described in the paper, Solar Cycle 14 (in dotted black) is predicted from the 5 sine functions that model the SSA reconstruction. (b) In red SSN Solar Cycles 13 to 24 (observed), in dotted black the reconstruction using SSA Solar Cycles 13 to 24 as described in the paper, Solar Cycle 25 predicted from the full data set is in green dots, and from half of the data set in black dots.

\section{Conclusion}

Finding whether the planets of the solar system, and in particular the Jovian planets, have an influence on planet Earth is currently undergoing a revival and has become the focus of renewed attention. The present paper deals with the prediction of the starting solar activity cycle, Solar Cycle 25. We propose that astronomical ephemeris, specifically the catalogs of aphelia of the four Jovian planets, can be used as evidence of a driving mechanism of variations in solar activity, represented by the series of sunspot numbers SSN from 1749 
Table 3 A selection (from Petrovay, 2020) of early forecasts for Solar Cycle 25, complemented with the result obtained in this paper using SSA. Abbreviations are SoDa $=$ Solar Dynamo, SFT $=$ Surface Flux Transport, AFT $=$ Advective Flux Transport.

\begin{tabular}{|c|c|c|c|c|}
\hline Category & Minimum & Maximum & $\begin{array}{l}\text { Peak } \\
\text { amplitude }\end{array}$ & References \\
\hline Internal precursors & 2019.9 & 2023.8 & $\begin{array}{l}175 \\
(154-202)\end{array}$ & Li, Feng, and Li (2015) \\
\hline \multicolumn{5}{|l|}{ External precursor } \\
\hline Polar precursor & & & $117 \pm 15$ & Table 1 here \\
\hline Polar precursor & & & $136 \pm 48$ & Pesnell and Schatten (2018) \\
\hline Helicity & & & 117 & Hawkes and Berger (2018) \\
\hline SoDA & & $2025.2 \pm 1.5$ & $120 \pm 39$ & $\begin{array}{l}\text { Based on Pesnell and } \\
\text { Schatten (2018) }\end{array}$ \\
\hline Rush-to-the-poles & 2019.4 & 2024.8 & 130 & Petrovay et al. (2018) \\
\hline \multicolumn{5}{|l|}{ Model-based: SFT } \\
\hline SFT & & & $124 \pm 31$ & Jiang and Cao (2018) \\
\hline AFT & 2020.9 & & 110 & Upton and Hathaway (2018) \\
\hline \multicolumn{5}{|l|}{ Model-based: dynamo } \\
\hline $2 \times 2 \mathrm{D}$ & $2020.5 \pm 0.12$ & $2027.2 \pm 1.0$ & $89_{-14}^{+29}$ & $\begin{array}{l}\text { Labonville, Charbonneau, and } \\
\text { Lemerle (2019) }\end{array}$ \\
\hline Truncated & 2019-2020 & $2024 \pm 1$ & $90 \pm 15$ & Kitiashvili (2016) \\
\hline \multicolumn{5}{|l|}{ Spectral } \\
\hline Wavelet decomposition tree & & 2023.4 & 132 & Rigozo et al. (2011) \\
\hline \multicolumn{5}{|l|}{ Attractor analysis } \\
\hline Simplex projection analysis & & $2024.0 \pm 0.6$ & $103 \pm 25$ & Singh and Bhargawa (2017) \\
\hline Simplex proj./time-delay & & $2023.2 \pm 1.1$ & $154 \pm 12$ & Sarp et al. (2018) \\
\hline \multicolumn{5}{|l|}{ Neural networks } \\
\hline Neuro-fuzzy & & 2022 & $90.7 \pm 8$ & Attia, Ismail, and Basurah (2013) \\
\hline Spatiotemporal & & $2022-2023$ & $57 \pm 17$ & $\begin{array}{l}\text { Covas, Peixinho, and } \\
\text { Fernandes (2019) }\end{array}$ \\
\hline Singular Spectrum Analysis & & $2026.2 \pm 1$ & $97.6 \pm 7.8$ & Courtillot et al. (this paper) \\
\hline
\end{tabular}

to 2020 (Appendix B). We use singular spectrum analysis (SSA) to associate components with similar periods in the ephemeris and SSN. We determine the transfer function between the two data sets, first with Jupiter only, then we improve the match in steps with the four Jovian planets and finally including commensurable periods of pairs and pairs of pairs of the Jovian planets (following for instance Mörth and Schlamminger, 1979). The transfer function can be applied to the ephemeris to predict future values of cycles. We have tested this with success with the hindcast of Solar Cycles 21 to 24 using only data preceding these cycles. We have also divided the full data set into two halves. Using the SSA method applied to Solar Cycles 1 to 13, we successfully "predict" the characteristics of Solar Cycle 14. Then, we use the second half of the SSN data (from Solar Cycle 14 to 24) to obtain another "prediction" of Solar Cycle 25. The shorter time series used results in (unacceptable) negative SSN values. This is interpreted as a failure to extract a proper trend from too short a data set, which does not allow a correct interpretation of the trend, as a result of ephemeris 
periods longer than the data interval of the truncated SSN series. But the trend is correctly recovered when the full-length series is used and the effect of the ephemeris of Uranus and Saturn is recognized. Figures 7 and 11 demonstrate the quality of the SSA model when one computes the sum of the SSA components with periods compatible with the revolution periods of Jovian planets and the periodicities of the ephemeris of commensurable pairs Jupiter/Saturn and Uranus/Neptune and pair of pairs (Jupiter/Saturn)/(Uranus/Neptune).

We conclude with a prediction of Solar Cycle 25 that can be compared to a dozen predictions by other authors (Petrovay, 2020): the maximum would occur in 2026.2 ( $\pm 1 \mathrm{yr}$ ) and reach an amplitude of $97.6( \pm 10)$, similar to that of Solar Cycle 24, therefore sketching a new "Modern minimum", following the Dalton and Gleissberg minima in the previous 200 years.

Although the exact mechanism for this influence of planets on the fluid envelopes of the Sun (photosphere) and also Earth (atmosphere and ocean) is still not fully understood, this influence is clearly apparent in the present paper. We have seen that for instance the long period trend that we extract with SSA in a time window of 200 years could in part be the signature of a long period ephemeris, such as periods linked to Neptune (165 yr; Table 1). We could include the terrestrial planets but expect smaller contributions (these could for instance have a bearing on features such as double maxima). In closing, we wish to emphasize the fact that the powerful SSA method of analysis of quasi-periodic oscillations is a central tool in this analysis. The reconstructed transfer functions that allow one to pass from the ephemeris to the sunspots should be applicable as long as the source (astronomical ephemeris) will stand and as long as we have included all effects from the relevant planets. The SSA reconstruction we propose can be used to predict beyond Solar Cycle 25, but may be degraded with time since oscillations longer than the data interval may have been missed.

Acknowledgements We thank Vladimir Kossobokov for comments and for help in calculating the statistics in this paper. We thank an anonymous reviewer for having insisted on points that needed clarification. IPG Contribution No 4185.

Disclosure of Potential Conflicts of Interest The authors declare that they have no conflicts of interest.

Publisher's Note Springer Nature remains neutral with regard to jurisdictional claims in published maps and institutional affiliations.

Open Access This article is licensed under a Creative Commons Attribution 4.0 International License, which permits use, sharing, adaptation, distribution and reproduction in any medium or format, as long as you give appropriate credit to the original author(s) and the source, provide a link to the Creative Commons licence, and indicate if changes were made. The images or other third party material in this article are included in the article's Creative Commons licence, unless indicated otherwise in a credit line to the material. If material is not included in the article's Creative Commons licence and your intended use is not permitted by statutory regulation or exceeds the permitted use, you will need to obtain permission directly from the copyright holder. To view a copy of this licence, visit http://creativecommons.org/licenses/by/4.0/.

\section{Appendix A}

We have shown in Figures 3 and 4 the Schwabe cycle, the aphelia of Jupiter, and the modulus and phase of the filter that allows one to go from one to the other. For the sake of brevity, we have shown only that one. In this appendix, we add the same information for the planets Uranus (Gleissberg cycle; Figures 13a and 13b) and Saturn (30 year cycle; Figures 14a and $14 \mathrm{~b}$ ). We could do the same for Neptune, but its period (164 years) can be interpreted on our too short time interval as a trend. 

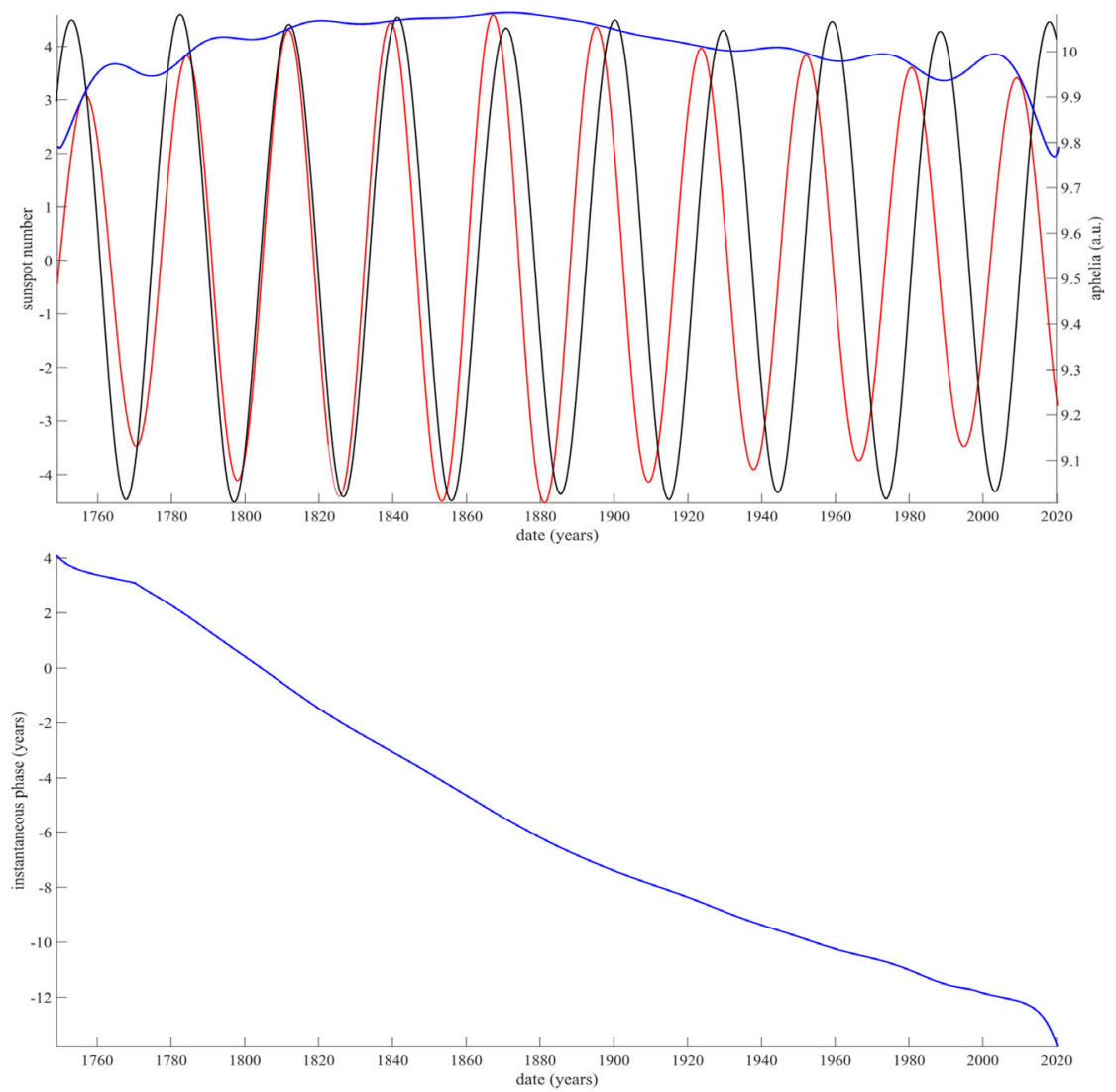

(a)

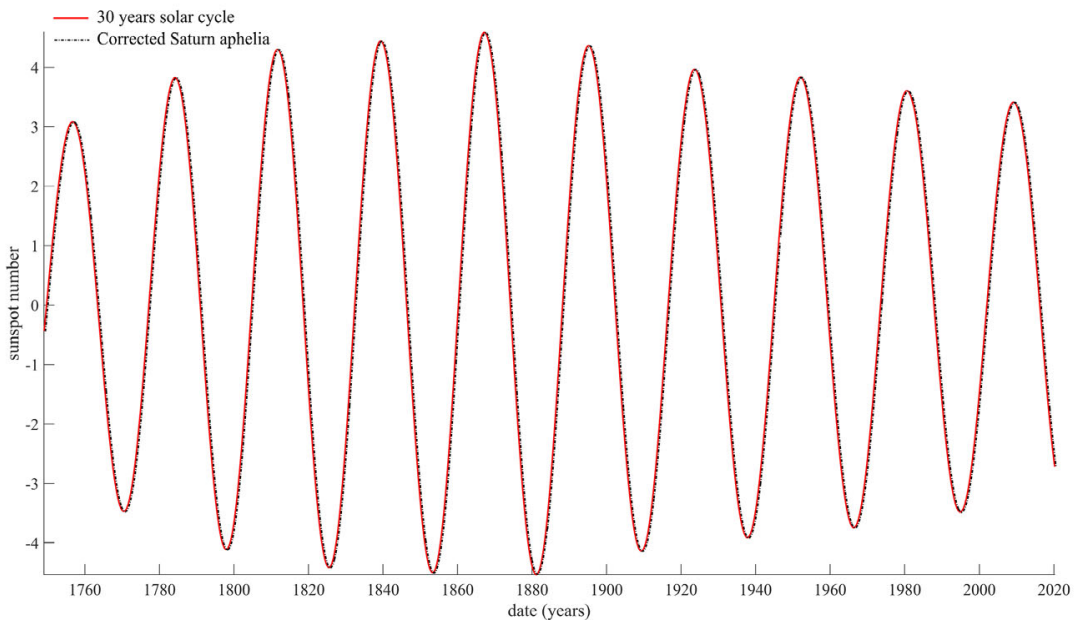

(b)

Figure 13 (a) Top, in black, the aphelia of Saturn and in red the 30-year cycle. In blue, the filter amplitude modulation (top) and the instantaneous phase difference between the two (bottom). (b) Superposition of the 30-year cycle extracted directly from SSN (red) and obtained by filtering the aphelia of Saturn (black, dotted). 

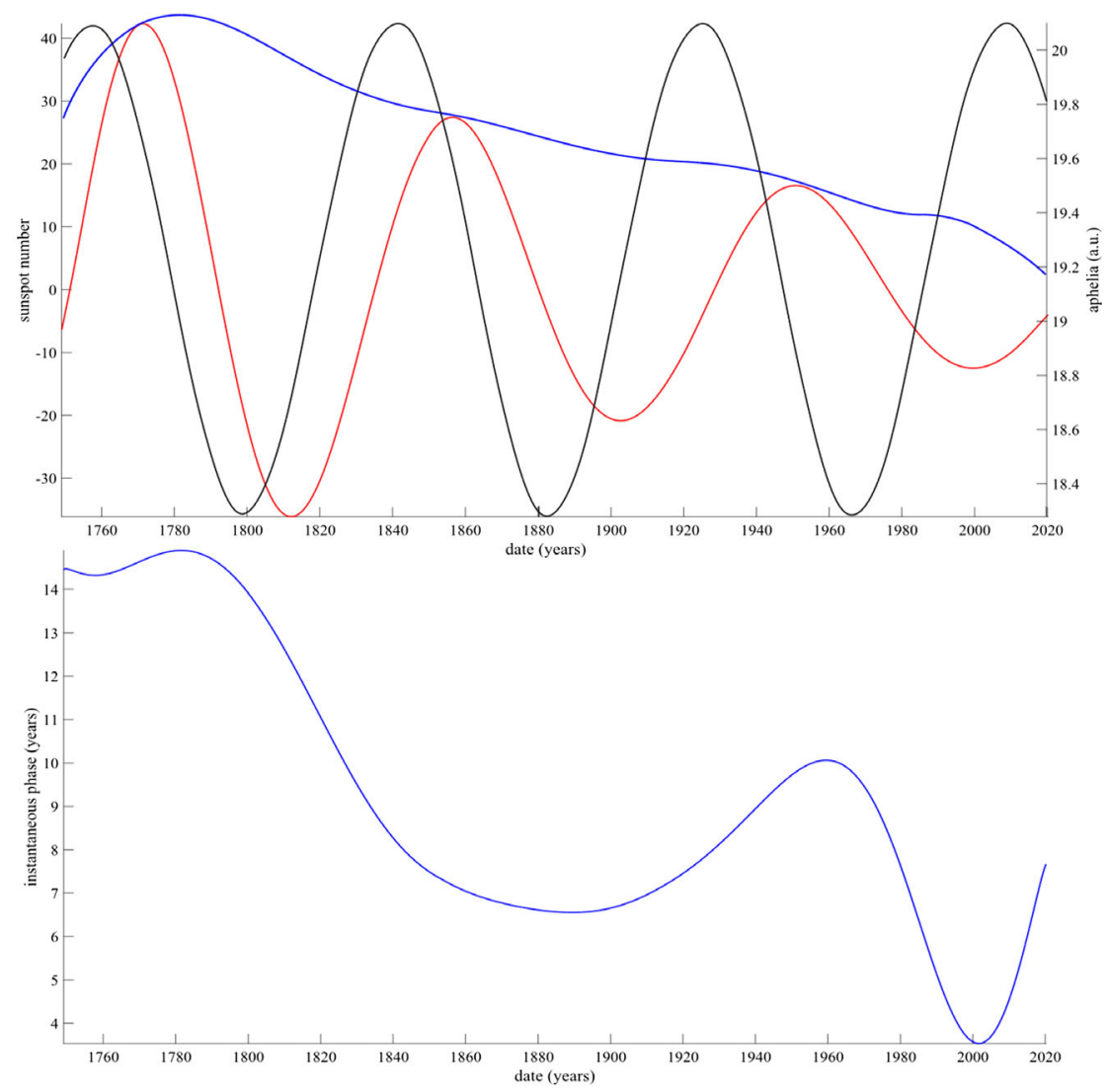

(a)

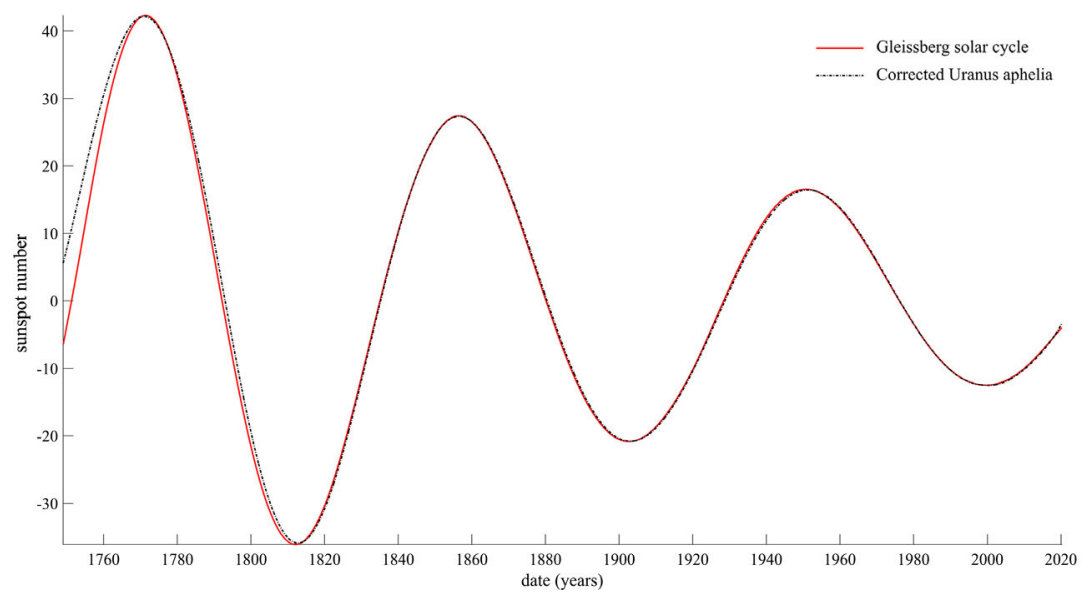

(b)

Figure 14 (a) Top, in black, the aphelia of Uranus and in red the Gleissberg cycle. In blue, the filter amplitude modulation (top) and its instantaneous phase (bottom). (b) Superposition of the Gleissberg cycle extracted directly from SSN (red) and obtained by filtering the aphelia of Uranus (black, dotted). 
We can make several significant remarks on these figures. First, we see that amplitudes and instantaneous phases are both regular and often monotonous, which ensures filter stability as a function of time (this might not be true for the rather strong phase variation seen in Figure 13a starting around 1960). Note that we work on real data using the Hilbert transform; the 90 -year period we wish to reproduce is long enough compared to the data interval to perturb the evaluation of the instantaneous phase.

However, this does not invalidate our approach, as shown in Figure 13b: there is an almost perfect superposition of the red (SSN data) and black dotted (filtered aphelia) curves. The oscillation at the end of the instantaneous phase plot will appear as long as the interval over which the time series SSN does not exceed eight times (Claerbout, 1976) the revolution period of Uranus (i.e. the Gleissberg cycle). This could be a problem if we want to construct a complete physical filter, i.e. estimate the time variations of all amplitudes and phases of all oscillations corresponding to aphelia or combinations of aphelia of planets. When phases and amplitudes are monotonous, the Kramer-Kronig relationships (that relate the real and imaginary parts of the filter via the Hilbert transform) ensure that the filter is stable (Claerbout, 1976; Papoulis, 1977). The stability of the techniques we use has been studied for instance by Vautard, Yiou, and Ghil (1992), Golyandina and Zhigljavsky (2013, Chapters 1 to 7). We have addressed it in studies of climate indices (Le Mouël, Lopes, and Courtillot, 2019a; see paragraph on Madden-Julian Oscillation) and surface temperatures (Le Mouël, Lopes, and Courtillot, 2020a; Appendix).

The second important point is that we always start with a positive phase difference between the aphelia and SSN; hence, under the hypothesis of a planetary forcing, the filters are causal. Being stable and causal, they are physical filters. To be precise, we have sinusoidal forcings that start and end at minus and plus infinity (as is the case for the bounds of the Fourier integral). Thus, between a sine and a cosine, which one is ahead? What does the instantaneous phase mean? We can propose a simple answer. We have calculated the instantaneous phase difference between planets and SSN; they are always in the same direction for all periods associated with the Jovian planets (and it would have been the same for the telluric planets). If there had been no underlying physical sense, nor causality, we should have found two statistical results: (i) instantaneous phase differences could have been negative from the start in 1749, (ii) phases could have been jagged, non-monotonous, not systematically decreasing.

We observe a coherency between the various periods (frequencies) that cannot be due to chance. In Le Mouël, Lopes, and Courtillot (2020a), we have already witnessed this coherency: the distribution of SSN component amplitudes as a function of period follows a Kolmogorov law with a $-5 / 3$ exponent. As a follow up of these studies, we could try to build a complete filter that would be the Sun's Green function, and tell us about the physical mechanism behind sunspots. This is left to future work.

\section{Appendix B}

Figure 15 displays from top to bottom the aphelion of Jupiter, its declination and right ascension from January 15, 1749 to March 15, 2020 (all with a period close to 11 years). When looking only at the aphelion, one follows the course of Jupiter around the Sun in the ecliptic plane. But, as is the case for all planets, Jupiter actually oscillates about the ecliptic according to its declination. This is shown as the bottom curve (aphelia multiplied by $\sin ($ declination $)$ ), thus with half the period of Jupiter, i.e. about 5.5 years. In the frame of a 

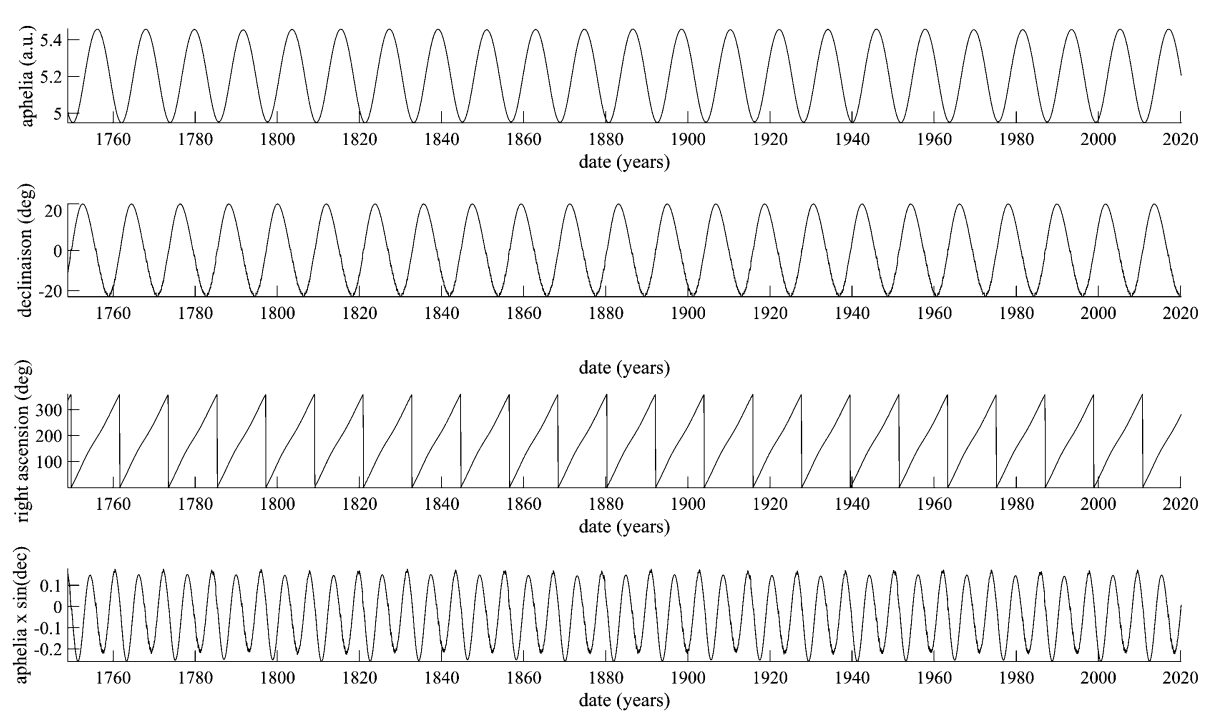

Figure 15 Monthly ephemeris of Jupiter from January 15, 1749 to March 15, 2020.

first order forcing of sunspots (the solar photosphere) by the Jovian planets, the half periods of the four planets $(5.5,15,42$, and 82 years) can be associated with the corresponding ephemeris (all can be accessed through the website of the Institut de Mécanique Céleste et de Calcul des Ephémérides (IMCCE, http://vo.imcce.fr/webservices/miriade/?forms).

\section{References}

Arlt, R., Vaquero, J.M.: 2020, Living Rev. Solar Phys. 17(1), 1. DOI.

Attia, A.F., Ismail, H.A., Basurah, H.M.: 2013, Astrophys. Space Sci. 344(5), 11. DOI.

Bhatt, N.J., Jain, R., Aggarwal, M.: 2009, Solar Phys. 260(1), 225. DOI.

Bushby, P.J., Tobias, S.M.: 2007, Astrophys. J. 661(2), 1289.

Cameron, R., Schüssler, M.: 2007, Astrophys. J. 659(1), 801.

Charbonneau, P.: 2013, Nature 493, 613. DOI.

Charbonneau, P.: 2014, Annu. Rev. Astron. Astrophys. 52, 251. DOI.

Claerbout, J.F.: 1976, Fundamentals of Geophysical Data Processing 274, McGraw-Hill, New York.

Clette, F., Lefèvre, L.: 2016, Solar Phys. 291, 2629. DOI.

Courtillot, V., Le Mouël, J.L.: 1976a, J. Geophys. Res. 81, 2941. DOI.

Courtillot, V., Le Mouël, J.L.: 1976b, Phys. Earth Planet. Inter. 12, 237. DOI.

Courtillot, V., Le Mouël, J.L., Kossobokov, V., Gibert, D., Lopes, F.: 2013, Atmos. Clim. Sci. 3, 364. DOI.

Covas, E., Peixinho, N., Fernandes, J.: 2019, Neural network forecast of the sunspot butterfly diagram. Solar Phys. 294(3), 24. DOI. arXiv.

Currie, R.G.: 1973, Astrophys. Space Sci. 21, 425. DOI.

Duhau, S.: 2003, Solar Phys. 213, 203. DOI.

Gleissberg, W.: 1939, Observatory 62, 158.

Golyandina, N., Zhigljavsky, A.: 2013, Singular Spectrum Analysis for Time Series, Springer, Berlin, 129 pp.

Hans Meier, A., Denkmayr, K., Weiss, P.: 1999, Solar Phys. 184, 213. DOI.

Hathaway, D.H., Wilson, R.M.: 2006, Geophys. Res. Lett. 33, L18101. DOI.

Hathaway, D.H., Wilson, R.M., Reichmann, E.J.: 1994, Solar Phys. 151, 177. DOI.

Hawkes, G., Berger, M.A.: 2018, Magnetic helicity as a predictor of the solar cycle. Solar Phys. 293, 109. DOI.

Jiang, J., Cao, J.: 2018, Predicting solar surface large-scale magnetic field of cycle 24. J. Atmos. Solar-Terr. Phys. 176, 34. DOI. arXiv. 
Jose, P.D.: 1965, Astrophys. J. 70, 193. DOI.

Kirkpatrick, S.C., Gelatt, D., Vecchi, M.P.: 1983, Science 220, 671. DOI.

Kitiashvili, I.N.: 2016, Data assimilation approach for forecast of solar activity cycles. Astrophys. J. 831(15). DOI.

Kossobokov, V.G., Le Mouël, J.L., Courtillot, V.: 2012, Solar Phys. 276, 383. DOI.

Kossobokov, V.G., Le Mouël, J.-L., Courtillot, V.: 2016, In: Jones, S.L. (ed.) Solar Flares: Investigations and Selected Research, Chapter 4, NOVA Science Publ. Physics Research and Technology Series, Hauppauge, New York, 81. ISBN: 978-1-53610-204-8.

Labonville, F., Charbonneau, P., Lemerle, A.: 2019, A dynamo-based forecast of solar cycle 25. Solar Phys. 294(6), 82. DOI.

Lantos, P., Richard, O.: 1998, Solar Phys. 182, 231. DOI.

Le Mouël, J.L., Lopes, F., Courtillot, V.: 2017, Solar Phys. 292, 43. DOI.

Le Mouël, J.L., Lopes, F., Courtillot, V.: 2019a, J. Geophys. Res. Space Phys. 124, 6403. DOI.

Le Mouël, J.L., Lopes, F., Courtillot, V.: 2019b, J. Geophys. Res., Atmos. 124, 2600. DOI.

Le Mouël, J.L., Lopes, F., Courtillot, V., Gibert, D.: 2019, Phys. Earth Planet. Inter. 292, 1. DOI.

Le Mouël, J.L., Lopes, F., Courtillot, V.: 2020a, Mon. Not. Roy. Astron. Soc. 492, 1416. DOI.

Le Mouël, J.L., Lopes, F., Courtillot, V.: 2020b, Earth Space Sci. 7(4). DOI.

Li, Y.: 1997, Predictions of the features for sunspot cycle 23. Solar Phys. 170(2), 437. DOI.

Li, R., Zhu, J.: 2013, Res. Astron. Astrophys. 13, 1118. DOI.

Li, K.J., Feng, W., Li, F.Y.: 2015, Predicting the maximum amplitude of solar cycle 25 and its timing. J. Atmos. Solar-Terr. Phys. 135, 72. DOI.

Lopes, F., Le Mouël, J.L., Gibert, D.: 2017, C. R. Géosci. 349, 159. DOI.

Malburet, J.: 2019, C. R. Géosci. 351, 351. DOI.

Maunder, E.W.: 1894, Knowledge: An Illustrated Magazine of Science 18, 173.

Maunder, E.W., Maunder, A.S.D.: 1905, Mon. Not. Roy. Astron. Soc. 65, 813.

Mayaud, P.N.: 1972, J. Geophys. Res. 77, 6870. DOI.

Mörth, H.T., Schlamminger, L.: 1979, In: Planetary Motion, Sunspots and Climate, Solar-Terrestrial Influences on Weather and Climate, Springer, Dordrecht, 193.

Mwitondi, K.S., Raeed, T.S., Yousif, A.E.: 2012, In: International Conference on Neural Information Processing, Springer, Berlin.

Okhlopkov, V.P.: 2016, Moscow Univ. Phys. Bull. 71, 440. DOI.

Papoulis, A.: 1977, Signal Analysis 191, McGraw-Hill, New York. 400 pp.

Pesnell, W.D.: 2008, Solar Phys. 252, 209. DOI.

Pesnell, W.D.: 2016, Space Weather 14, 10. DOI.

Pesnell, W.D., Schatten, K.H.: 2018, An early prediction of the amplitude of solar cycle 25. Solar Phys. 293, 112. DOI.

Petrovay, K.: 2020, Living Rev. Solar Phys. 17, 1. DOI.

Rekapalli, R., Tiwari, R.K.: 2020, Pure Appl. Geophys. 177, 5469. DOI.

Rigozo, N.R., Souza Echer, M.P., Evangelista, H., Nordemann, D.J.R., Echer, E.: 2011, Prediction of sunspot number amplitude and solar cycle length for cycles 24 and 25. J. Atmos. Solar-Terr. Phys. 73(11-12), 1294. DOI.

Petrovay, K., Nagy, M., Gerják, T., Juhász, L.: 2018, Precursors of an upcoming solar cycle at high latitudes from coronal green line data. J. Atmos. Solar-Terr. Phys. 176, 15. DOI. arXiv.

Sarp, V., Kilcik, A., Yurchyshyn, V., Rozelot, J.P., Ozguc, A.: 2018, Prediction of solar cycle 25: a non-linear approach. Mon. Not. Roy. Astron. Soc.. 481, 2981. DOI.

Scafetta, N.: 2020, Solar Phys. 295, 1. DOI.

Schatten, K.H., Scherrer, P.H., Svalgaard, L., Wilcox, J.M.: 1978, Geophys. Res. Lett. 5, 411. DOI.

Singh, A.K., Bhargawa, A.: 2017, An early prediction of 25th solar cycle using Hurst exponent. Astrophys. Space Sci. 362, 199. DOI.

Stefani, F., Gieseke, A., Weier, T.: 2019, Solar Phys. 294, 60. DOI.

Stefani, F., Beer, J., Giesecke, A., Gloaguen, T., Seilmayer, M., Stepanov, R., Weier, T.: 2020, Astron. Notes 341, 1. DOI.

Svalgaard, L., Cliver, E.W., Kamide, Y.: 2005, Geophys. Res. Lett. 32, L01104. DOI.

Upton, L.A., Hathaway, D.H.: 2018, An updated solar cycle 25 prediction with AFT: the modern minimum. Geophys. Res. Lett. 45, 8091. DOI. arXiv.

Vaquero, J.M., Svalgaard, V.M.S., Carrasco, F., Clette, L., Lefèvre, M.C., Gallego, R., Arlt, R., Aparicio, A.J.P., Richard, J.G., Howe, R.: 2016, Solar Phys. 291, 3061. DOI.

Vautard, R., Yiou, P., Ghil, M.: 1992, Physica D 58, 95. DOI.

Whitehouse, D.: 2020, The Next Solar Cycle and Why It Matters for Climate, Note 22, The Global Warming Policy Foundation, London. https://www.thegwpf.org/content/uploads/2020/04/SolarCycle25.pdf.

Wilson, R.M.: 1988, J. Geophys. Res. Space Phys. 93, 10011. DOI.

Wolf, R.: 1852, Mitteilungen der Naturforschenden Gesellschaft in Bern 255, 249 (in German). 